\title{
Theoretical Limits for Estimation of Periodic Movements in Pulse-Based UWB Systems
}

\author{
Sinan Gezici, Member, IEEE
}

\begin{abstract}
In this paper, Cramer-Rao lower bounds (CRLBs) for estimation of signal parameters related to periodically moving objects in pulse-based ultra-wideband (UWB) systems are presented. The results also apply to estimation of vital parameters, such as respiration rate, using $U W B$ signals. In addition to obtaining the CRLBs, suboptimal estimation algorithms are also presented. First, a single-path channel with additive white Gaussian noise is considered, and closed-form CRLB expressions are obtained for sinusoidal object movements. Also, a two-step suboptimal algorithm is proposed, which is based on time delay estimation via matched filtering followed by least-squares estimation, and its asymptotic optimality property is shown in the limit of certain system parameters. Then, a multipath environment is considered, and exact and approximate CRLB expressions are derived. Moreover, suboptimal schemes for parameter estimation are studied. Simulation studies are performed for the estimation of respiration rates in order to evaluate the lower bounds and performance of the suboptimal algorithms for realistic system parameters.
\end{abstract}

Index Terms-Cramer-Rao lower bound (CRLB), estimation theory, impulse radio (IR), least-squares (LS) estimation, matched filtering, ultra-wideband (UWB).

\section{INTRODUCTION}

A FTER the US Federal Communications Commission (FCC) approved the limited use of ultra-wideband (UWB) technology in February 2002 [1], communications and imaging systems that employ UWB signals have drawn considerable attention. A UWB signal is defined to be one that possesses an absolute bandwidth larger than $500 \mathrm{MHz}$ or a fractional (relative) bandwidth larger than $20 \%$. The large bandwidth of UWB signals not only facilitates high-speed data transmission, but also results in high time resolution, which is crucial for precise ranging and location estimation [2], [3].

Commonly, impulse radio (IR) systems, which transmit very short duration pulses with a low duty cycle, are employed to implement UWB systems ([4]-[7]). In an IR communications system, a train of pulses is sent and information is usually conveyed by the positions or the amplitudes of the pulses, which correspond to pulse position modulation (PPM) and pulse amplitude modulation (PAM), respectively. In multi-user environments, in order to prevent catastrophic collisions among pulses of different users and thus provide robustness against multiple

Manuscript received November 30, 2006; revised July 6, 2007. This work was presented in part at the IEEE International Conference on Communications, Glasgow, Scotland, June 2007.

The author is with the Department of Electrical and Electronics Engineering, Bilkent University, Bilkent, Ankara 06800, Turkey (e-mail: gezici@ee.bilkent. edu.tr).

Color versions of one or more of the figures in this paper are available online at http://ieeexplore.ieee.org.

Digital Object Identifier 10.1109/JSTSP.2007.906645 access interference, each information symbol is represented by a sequence of pulses and the positions of the pulses within that sequence are determined by a pseudorandom time-hopping (TH) sequence specific to each user [4].

In addition to communication applications, UWB systems have also been considered for radar/imaging applications, such as medical imaging [8], [9], ground penetrating radars [10], through-wall imaging [11], [12], and surveillance systems [13]. In this paper, we consider the problem of estimating the parameters of a moving object in a given environment by means of pulse-based UWB signals, where the main focus is to estimate the frequency of a periodically moving object. The problem framework also covers the case in which there is no object in the environment; hence, both detection of periodic movements and estimation of movement parameters are performed. From this viewpoint, the problem can be considered in a UWB radar framework. The main advantages of UWB radars [14] compared to conventional narrowband radars [15]-[17] are high accuracy, low transmission power, and good penetration capability, which facilitates through-the-wall applications [11], [12], [18].

Parameter estimation problem for moving objects bears some resemblance to channel estimation problem as well [19]-[22], since information on movement parameters can be obtained by observing the time variation of the signal delays corresponding to reflections from the object. However, in the movement estimation problem, the main parameters to be estimated are related to the time varying multipath components only, and the other channel parameters such as the channel coefficients and delays (corresponding to other multipath components) are all considered as nuisance parameters. On the other hand, the channel estimation problem [19], [20], [22] considers channel coefficients and delays as the main parameters to be estimated. In addition, the observation interval in a movement estimation problem is considerably larger than that in a channel estimation problem. For that reason, multipath components are assumed to be static for the latter case. On the other hand, in a movement-estimation scenario, multiple pulse bursts are employed, where the delays between consecutive bursts are comparable to the period of the movement in the environment [23].

Estimation of signal parameters related to a periodically moving object has applications in vital signs monitoring, such as respiration rate estimation, since UWB pulses have sufficiently high time resolution to detect movements of a human chest, and even of a heart [23]-[27]. Use of UWB signals for vital signal monitoring facilitates many medical applications, such as respiratory activity monitoring in intensive care units, cardiac monitoring, sudden infant death syndrome detection and arterial-wall motion monitoring [24]. In addition to noninvasive monitoring applications, estimation of vital 


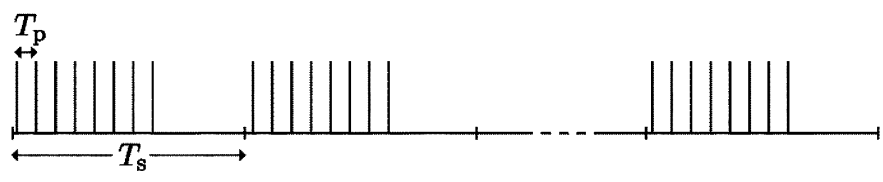

Fig. 1. Transmitted signal structure for estimation of signal parameters related to object movement. Pulses in each burst are used to improve SNR at the receiver, and different bursts give information about movement parameters.

(medical) signal parameters can be very important in many other scenarios, such as searching people under debris after an earthquake and through-the-wall health monitoring of hostages [23].

Previous studies on (periodic) movement estimation via UWB signals were usually focused on medical or security applications. In [24], possible medical applications of UWB signals are studied, and their penetration and reflection properties are investigated. In [25] and [26], estimation of respiration and heartbeat rates via UWB signals is studied experimentally. A mathematical framework for estimation of medical signal parameters is established in [23], which investigates pulse-based UWB signals and filtering approaches for estimation of respiration and heartbeat rates. In [18], a UWB radar is designed to determine the location of moving people behind walls.

Although UWB parameter estimation problem for periodically moving objects was studied for medical and security applications [18], [23]-[26], no studies have considered the theoretical limits and asymptotically optimal solutions for this parameter estimation problem. In this paper, we derive Cramer-Rao lower bounds (CRLBs) for estimation of signal parameters related to a periodically ${ }^{1}$ moving object, and study maximum likelihood and suboptimal estimation algorithms for single-path and multipath channel conditions. We also numerically evaluate CRLBs for a practical respiration rate estimation scenario, and compare the performance of the suboptimal algorithms with the CRLBs.

The remainder of the paper is organized as follows. In Section II, the signal model used for parameter estimation is presented. In Section III, the maximum likelihood (ML) solution is obtained and generic CRLB expressions are derived. Closed-form expressions for theoretical limits and an asymptotically optimal solution are studied in Section IV for single path additive white Gaussian noise (AWGN) channels. Then, in Section V, multipath channels are studied and theoretical limits and suboptimal algorithms are investigated. Finally, numerical examples and simulation results are presented in Section VI, followed by the concluding remarks in Section VII.

\section{SIGNAL MODEL}

The transmitted signal consists of a sequence of pulse bursts as shown in Fig. 1, and is expressed as follows:

$$
s(t)=\frac{1}{\sqrt{N}} \sum_{k=0}^{N-1} w_{\mathrm{tx}}\left(t-k T_{\mathrm{s}}\right)
$$

\footnotetext{
${ }^{1}$ The derivations are first obtained for any generic displacement function, not necessarily periodic. Then, simpler expressions are obtained for sinusoidal movements.
}

where $N$ is the number of bursts, $T_{\mathrm{s}}$ is the burst period, and $w_{\mathrm{tx}}(t)$ is a burst of pulses, which consists of $M$ pulses and is expressed as

$$
w_{\mathrm{tx}}(t)=\sum_{j=0}^{M-1} p_{\mathrm{tx}}\left(t-j T_{\mathrm{p}}\right)
$$

with $p_{\mathrm{tx}}(t)$ denoting the transmitted pulse and $T_{\mathrm{p}}$ being the interval between consecutive pulses. It is assumed that $T_{\mathrm{p}}>T_{\mathrm{w}}$, where $T_{\mathrm{w}}$ denotes the width of $p_{\mathrm{tx}}(t)$.

The burst of pulses in (1) is aimed at an object being monitored, and the reflections are collected by a receiver. As in typical pulse-based radar applications [28], pulses in each burst are employed to obtain a reliable channel profile, and comparison of channel profiles obtained from consecutive bursts is used to estimate certain parameters of the object in the environment.

The received signal can be expressed as

$$
r(t)=g_{\boldsymbol{\theta}}(t)+\sigma n(t)
$$

where $g_{\boldsymbol{\theta}}(t)$ represents the signal part, with $\boldsymbol{\theta}$ representing the vector of all unknown signal parameters, and $n(t)$ denotes zeromean white Gaussian noise with unit spectral density. It is assumed that the average noise power (or $\sigma^{2}$ ) is known. From the transmitted signal model in (1), the signal part $g_{\boldsymbol{\theta}}(t)$ in (3) can be expressed as ${ }^{2}$

$$
\begin{array}{r}
g_{\boldsymbol{\theta}}(t)=\frac{1}{\sqrt{N}} \sum_{k=0}^{N-1}\left(\sum_{l \in \mathcal{L}-\{b\}} \alpha_{l} w_{l}\left(t-k T_{\mathrm{S}}-\tau_{l}\right)\right. \\
\left.\quad+\alpha_{b} w_{b}\left(t-k T_{\mathrm{S}}-\tau_{b}-h_{k}(\boldsymbol{\lambda})\right)\right)
\end{array}
$$

where $w_{l}(t)$ is the received pulse burst via the $l$ th multipath component, $w_{l}(t)=\sum_{j=0}^{M-1} p_{\text {rx }}^{(l)}\left(t-j T_{\mathrm{p}}\right)$, with $p_{\text {rx }}^{(l)}(t)$ denoting the received pulse via the $l$ th path, $\mathcal{L}=\{1, \ldots, L\}$ represents the set of indices for multipath components, $\alpha_{l}$ and $\tau_{l}$ are, respectively, the channel coefficient and the delay for the $l$ th multipath component $(l \in \mathcal{L})$, and $h_{k}(\boldsymbol{\lambda})$ represents the time displacement function induced by the periodically moving object in the environment with $\boldsymbol{\lambda}=\left[\lambda_{1} \cdots \lambda_{K}\right]$ denoting the unknown signal parameters related to the displacement function. Note that the channel coefficients and delays for all multipath components except the $b$ th one are assumed to be constant during the observation of $N$ signal bursts [23], [27] and that the $b$ th multipath component represents the reflection from the object (e.g., a human chest), which results in a variable path delay among different signal bursts. Also note that for the received signal model in (3) and (4), it is assumed that there is only one periodically moving object in the environment.

The unknown parameter vector $\boldsymbol{\theta}$ in (4) consists of unknown signal parameters related to $h_{k}(\boldsymbol{\lambda})$, and the parameters related to the multipath channel, which can be expressed as

$$
\boldsymbol{\theta}=\left[\begin{array}{lll}
\boldsymbol{\lambda} & \boldsymbol{\tau} & \boldsymbol{\alpha}
\end{array}\right]
$$

with $\boldsymbol{\tau}=\left[\tau_{1} \cdots \tau_{L}\right]$ and $\boldsymbol{\alpha}=\left[\alpha_{1} \cdots \alpha_{L}\right]$. Without loss of generality, the multipath components are assumed to be ordered; i.e., $\tau_{1}<\tau_{2}<\cdots<\tau_{L}$.

$$
{ }^{2} \mathcal{L}-\{b\} \text { consists of the elements of set } \mathcal{L} \text { that are different from } b .
$$


It is also assumed that $T_{\mathrm{s}}>\left(\tau_{L}-\tau_{1}\right)+(M-1) T_{\mathrm{p}}+T_{\mathrm{w}}+$ $\max \left\{h_{k}(\boldsymbol{\lambda})\right\}-\min \left\{h_{k}(\boldsymbol{\lambda})\right\}$, so that there is no interference between consecutive pulse bursts, which is usually the case in practical situations $^{3}$ [23].

\section{ML SOLUTION AND GENERIC CRLB EXPRESSIONS}

When the signal $r(t)$ in (3) is observed over a time interval $[0, T]$, with $T=N T_{\mathrm{s}}$, the $\log$-likelihood function of $\boldsymbol{\theta}$ can be expressed as

$$
\Lambda(\boldsymbol{\theta})=c-\frac{1}{2 \sigma^{2}} \int_{0}^{T}\left|r(t)-g_{\boldsymbol{\theta}}(t)\right|^{2} d t
$$

where $c$ denotes a constant independent of $\boldsymbol{\theta}$.

Considering real signals, the ML solution can be obtained from (6) as

$$
\hat{\boldsymbol{\theta}}_{\mathrm{ML}}=\underset{\boldsymbol{\theta}}{\operatorname{argmax}} \int_{0}^{T}\left[2 r(t) g_{\boldsymbol{\theta}}(t)-g_{\boldsymbol{\theta}}^{2}(t)\right] d t
$$

which requires exhaustive search over the parameter space for $\boldsymbol{\theta}$, which is impractical in most cases.

From (6), the components of the Fisher information matrix (FIM) I can be obtained as follows:

$$
[\mathbf{I}]_{i i}=E\left\{\left(\frac{\partial \Lambda(\boldsymbol{\theta})}{\partial \theta_{i}}\right)^{2}\right\}=\frac{1}{\sigma^{2}} \int_{0}^{T}\left(\frac{\partial g_{\boldsymbol{\theta}}(t)}{\partial \theta_{i}}\right)^{2} d t
$$

for $i=1, \ldots, 2 L+K$ and

$$
[\mathbf{I}]_{i j}=E\left\{\frac{\partial \Lambda(\boldsymbol{\theta})}{\partial \theta_{i}} \frac{\partial \Lambda(\boldsymbol{\theta})}{\partial \theta_{j}}\right\}=\frac{1}{\sigma^{2}} \int_{0}^{T} \frac{\partial g_{\boldsymbol{\theta}}(t)}{\partial \theta_{i}} \frac{\partial g_{\boldsymbol{\theta}}(t)}{\partial \theta_{j}} d t
$$

for $i \neq j$, where $\theta_{i}$ represents the $i$ th element of the parameter vector $\boldsymbol{\theta}$ in (5).

From (8) and (9), explicit exact and approximate expressions for the theoretical limits of signal parameter estimation are derived in Sections IV and V.

\section{SingLe-PATH SCENARIO}

\section{A. General Theory}

For an AWGN channel model with a single path component, the signal part $g_{\boldsymbol{\theta}}(t)$ in (4) can be expressed as ${ }^{4}$

$$
g_{\boldsymbol{\lambda}}(t)=\frac{1}{\sqrt{N}} \sum_{k=0}^{N-1} w_{b}\left(t-k T_{\mathrm{s}}-h_{k}(\boldsymbol{\lambda})\right)
$$

where $\boldsymbol{\theta}$ in (5) becomes equal to $\boldsymbol{\lambda}$ in this case.

Although the signal model in (10) is not very realistic for wideband pulse-based systems, the analysis for the single-path scenario provides important intuition for the parameter estimation problem, and the main ideas in the analysis can be employed to obtain results for a multipath scenario (Section V). Also, this model gets more accurate when directional antennas are used

\footnotetext{
${ }^{3}$ Note that, for the simplicity of the expressions, we assume the same pulsewidth $T_{\mathrm{w}}$ for all UWB pulses $p_{\mathrm{rx}}^{(l)}(t) l \in \mathcal{L}$. The analysis in the paper can be extended straightforwardly to the case in which UWB pulses received via different multipath components have different pulsewidths.

${ }^{4}$ In this case, it is assumed that the receiver is synchronized to the incoming signal. This assumption will be removed in Section V.
}

for transmission and reception, and/or an efficient clutter removal algorithm [28] is applied before parameter estimation. Of course, the theoretical limits on the accuracy of parameter estimation obtained using the single path model in this section provides a lower bound for the multipath case as well, since more nuisance parameters exist for the latter. Note, however, that the lower bound for the single-path case may not be very tight for the multipath scenario.

From (10), (8) and (9) can be expressed, after some manipulation, as

$$
\begin{aligned}
{\left[\mathbf{I}_{\mathrm{SP}}\right]_{i i} } & =\frac{\tilde{E}_{b}}{N \sigma^{2}} \sum_{k=0}^{N-1}\left(\frac{\partial h_{k}(\boldsymbol{\lambda})}{\partial \lambda_{i}}\right)^{2} \\
{\left[\mathbf{I}_{\mathrm{SP}}\right]_{i j} } & =\frac{\tilde{E}_{b}}{N \sigma^{2}} \sum_{k=0}^{N-1} \frac{\partial h_{k}(\boldsymbol{\lambda})}{\partial \lambda_{i}} \frac{\partial h_{k}(\boldsymbol{\lambda})}{\partial \lambda_{j}}
\end{aligned}
$$

for $i, j \in\{1, \ldots, K\}$ and $i \neq j$, where the subscript SP denotes the single path scenario, and $\tilde{E}_{b}$ is the energy of the first derivative of the pulse burst $w_{b}(t)$ (denoted as $w_{b}^{\prime}(t)$ ); i.e.,

$$
\tilde{E}_{b}=\int_{-\infty}^{\infty}\left(w_{b}^{\prime}(t)\right)^{2} d t=M \int_{-\infty}^{\infty}\left[\left(p_{\mathrm{rx}}^{(b)}(t)\right)^{\prime}\right]^{2} d t .
$$

Note that from the definition of the effective bandwidth of $w_{b}(t)$, i.e.,

$$
\beta=\left(\frac{\int_{-\infty}^{\infty} f^{2}\left|W_{b}(f)\right|^{2} d f}{\int_{-\infty}^{\infty}\left|W_{b}(f)\right|^{2} d f}\right)^{1 / 2}
$$

with $W_{b}(f)$ denoting the Fourier transform of $w_{b}(t), \tilde{E}_{b}$ in (13) can be expressed as

$$
\tilde{E}_{b}=4 \pi^{2} E_{b} \beta^{2}
$$

where $E_{b}$ is the energy of $w_{b}(t)$.

The CRLB for the covariance of an unbiased estimate of $\boldsymbol{\lambda}$ can be expressed as [29]

$$
\operatorname{Cov}\{\hat{\boldsymbol{\lambda}}\} \geq \mathbf{I}_{\mathrm{SP}}^{-1}
$$

where $\mathbf{B}_{1} \geq \mathbf{B}_{2}$ means that $\mathbf{B}_{1}-\mathbf{B}_{2}$ is nonnegative definite.

Let $\lambda_{1}=f$ denote the rate (frequency) parameter to be estimated (e.g., respiration rate). Then, the CRLB for estimating $f$ can be stated as

$$
\operatorname{Var}\{\hat{f}\} \geq\left[\mathbf{I}_{\mathrm{SP}}^{-1}\right]_{11}
$$

where $[\mathbf{B}]_{i j}$ denotes the element of matrix $\mathbf{B}$ in the $i$ th row and $j$ th column.

\section{B. Sinusoidal Displacement Function}

For the remainder of this section, the displacement function $h_{k}(\boldsymbol{\lambda})$ is modeled to have three unknown parameters; frequency $f$, phase $\phi$ and time shift $A$ corresponding to maximum displacement from nominal position. In other words, $\lambda=\left[\begin{array}{lll}f & \phi & A\end{array}\right]$. The main parameter of interest is the frequency of the displacement function, which corresponds, for example, to respiration rate of a human. The phase parameter is another unknown since the initial position of the object 
(e.g., chest cavity) is not known by the receiver. Also, the time shift for the maximum displacement amount from the nominal object position, $A$, is commonly unknown. Since the frequency is the main parameter of interest, the phase and the maximum displacement parameters, $\phi$ and $A$, are treated as nuisance parameters.

For the special case of a displacement function modeled by a sinusoidal function [23], [27], $h_{k}(\boldsymbol{\lambda})$ is given by

$$
h_{k}(\boldsymbol{\lambda})=A \sin \left(2 \pi f k T_{\mathrm{s}}+\phi\right) \text {. }
$$

From (18), (11) and (12), the CRLB in (17) can be obtained as 5

$$
\begin{aligned}
{\left[\mathbf{I}_{\mathrm{SP}}^{-1}\right]_{11}=} & \frac{N \sigma^{2}}{2 \pi^{2} T_{\mathrm{s}}^{2} A^{2} \tilde{E}}\left(X_{22} X_{33}-X_{23}^{2}\right) \\
& \times\left[X_{11}\left(X_{22} X_{33}-X_{23}^{2}\right)\right. \\
& \quad-X_{12}\left(X_{12} X_{33}-X_{23} X_{13}\right) \\
& \left.\quad+X_{13}\left(X_{12} X_{23}-X_{22} X_{13}\right)\right]^{-1}
\end{aligned}
$$

where

$$
\begin{aligned}
& X_{11}=\sum_{k=0}^{N-1} k^{2}\left(1+\cos \psi_{k}\right), \quad X_{22}=\sum_{k=0}^{N-1}\left(1+\cos \psi_{k}\right) \\
& X_{33}=\sum_{k=0}^{N-1}\left(1-\cos \psi_{k}\right), \quad X_{12}=\sum_{k=0}^{N-1} k\left(1+\cos \psi_{k}\right) \\
& X_{13}=\sum_{k=0}^{N-1} k \sin \psi_{k}, \quad X_{23}=\sum_{k=0}^{N-1} \sin \psi_{k}
\end{aligned}
$$

with $\psi_{k}=4 \pi f k T_{\mathrm{s}}+2 \phi$.

Although the exact CRLB can be obtained from (19) and (20), a simpler and more intuitive expression can be obtained in the case of a large number of received pulse bursts $N$. The following result can be used to obtain an approximate CRLB expression for large $N$.

Proposition 1: Assume that $T_{\mathrm{s}}<1 /(4 f)$. Then, as $N \longrightarrow$ $\infty$, the CRLB for $f$ multiplied by $N^{2}$ is expressed as

$$
\lim _{N \rightarrow \infty} N^{2}\left[\mathbf{I}_{\mathrm{SP}}^{-1}\right]_{11}=\frac{6 \sigma^{2}}{\pi^{2} \tilde{E} A^{2} T_{\mathrm{S}}^{2}} .
$$

\section{Proof: See Appendix A.}

Note that, in practice, $1 / T_{\mathrm{s}}$ is selected to be larger than twice the maximum possible frequency of the displacement function. For example, in [23], for respiratory-rate estimation, $T_{\mathrm{S}}$ is chosen to be 0.1 second, which corresponds to $1 / T_{\mathrm{S}}$ being about 10 times the common value for $f$. Therefore, the assumption of $T_{\mathrm{s}}<1 /(4 f)$ is valid in most practical situations.

For large $N$, the CRLB for $f$ can be approximated, from (21), as

$$
\left[\mathbf{I}_{\mathrm{SP}}^{-1}\right]_{11} \approx \frac{6 \sigma^{2}}{\pi^{2} \tilde{E} A^{2} T_{\mathrm{S}}^{2} N^{2}} \approx \frac{0.0154}{\operatorname{SNR} \beta^{2} A^{2} T_{\mathrm{S}}^{2} N^{2}}
$$

where the second expression is obtained from (15), with SNR = $E / \sigma^{2}$.

${ }^{5}$ For the remainder of this section, the subscript $b$ is dropped for convenience.

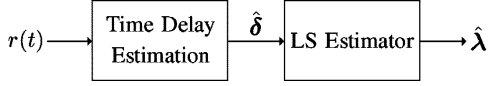

Fig. 2. Two-step suboptimal solution for estimation signal parameters.

From (22), it is observed that as the maximum time displacement amount $A$ or the number of bursts $N$ increases, the minimum achievable variance decreases with the square of these terms. In addition, $\tilde{E} / \sigma^{2}$ (or, the product of the SNR and the square of the effective bandwidth) is inversely proportional to the CRLB. It is also important to note from (22) that the approximate expression for the minimum achievable accuracy is independent of the true frequency and phase values. In other words, for any frequency satisfying $f<1 /\left(4 T_{\mathrm{s}}\right)$ and any phase, the achievable accuracy is the same for given $A, T\left(T=N T_{\mathrm{s}}\right)$ and $\tilde{E} / \sigma^{2}$ values.

Comparison of (22) with the CRLB for estimating the frequency of a sinusoidal signal (single tone) in AWGN (e.g., [33, pp. 56-57]) reveals that the CRLB in (22) is inversely proportional to the square of the number of bursts $N$ (or the observation interval), whereas the CRLB for frequency estimation of a single tone is inversely proportional to the cube of the number of samples (or the observation interval). In addition, there is an additional dependence on the effective bandwidth in (22) since the accuracy of frequency estimation depends on the accuracy of time-delay estimation for the signal model given by (10) and (18), which is affected by the effective bandwidth of the pulses.

\section{Suboptimal Solution}

Now that the best achievable accuracy for frequency estimation has been obtained, the problem of obtaining a practical solution that (asymptotically) achieves this best accuracy is considered next. Note that the ML solution in (7) is an optimal solution; however, it requires correlation of the received signal over the observation interval $[0, T]$ with the template signal $g_{\boldsymbol{\lambda}}(t)$ for various values of the parameter $\boldsymbol{\lambda}$, which has prohibitive complexity for practical implementations.

Instead of the ML solution in (7), the solution described in Fig. 2 is proposed. Namely, from $M$ pulses in each burst, the delay (displacement) of the signal in that burst interval is estimated. Let $\delta_{k} \triangleq h_{k}(\boldsymbol{\lambda})$ denote the delay of the $k$ th burst. Note that $\delta_{k}$ can be estimated by the conventional TOA estimation algorithm based on matched filter or correlator outputs [15], [30].

Let $\hat{\boldsymbol{\delta}}=\left[\begin{array}{llll}\hat{\delta}_{0} & \hat{\delta}_{1} & \cdots & \hat{\delta}_{N-1}\end{array}\right]$ denote the delay estimates obtained from matched filter processing. Then, these delay estimates are used by a least-squares (LS) estimator as shown in Fig. 2 to estimate the unknown parameters

$$
\hat{\boldsymbol{\lambda}}=\underset{\boldsymbol{\lambda}}{\operatorname{argmin}}\left\{\sum_{k=0}^{N-1}\left(\hat{\delta}_{k}-h_{k}(\boldsymbol{\lambda})\right)^{2}\right\} .
$$

Note that this two-step algorithm is considerably simpler and more practical than the ML solution in (7) since it does not have to perform correlations over the whole observation interval for all different values of $\boldsymbol{\lambda}$. Instead, it first performs a search over a single dimension, namely time delay, using the matched filter 
outputs for each given burst. Then, it uses these time delay estimates from different bursts for the LS estimation of the desired signal parameter(s).

The proposed two-step algorithm provides a suboptimal solution in general. However, it will be shown in the remainder of this section that the performance of this two-step algorithm gets very close to the CRLB given by (11)-(16), under certain conditions. Towards this end, the following result is considered first:

Lemma 1 [31], [32]: Consider the signal model

$$
\tilde{r}(t)=\frac{1}{\sqrt{N}} w\left(t-\delta_{k}\right)+\sigma n(t), \quad t \in\left[0, T_{\mathrm{s}}\right]
$$

where $n(t)$ is zero-mean white Gaussian noise with unit spectral density, and $\delta_{k}$ is the time delay to be estimated. If $\delta_{k}$ is estimated at a matched-filter output with large SNR. $\beta^{2}$, where $\beta^{2}=\int f^{2}|W(f)|^{2} d f / \int|W(f)|^{2} d f$, the estimate can be modeled as

$$
\hat{\delta}_{k}=\delta_{k}+n_{k}
$$

where $n_{k}$ is a Gaussian random variable $\mathcal{N}\left(0, \sigma_{0}^{2}\right)$ with $\sigma_{0}^{2}=$ $1 /\left(4 \pi^{2} \beta^{2} \mathrm{SNR}\right)$.

In other words, Lemma 1 states that for signals with large SNR and/or effective bandwidth $\beta$, the error of the conventional time delay estimation can be modeled by a zero mean Gaussian random variable. Note that the result of Lemma 1 basically follows from the fact that the matched filter (correlator) is an ML estimate for the time delay, hence provides an unbiased estimate and achieves the CRLB asymptotically [29].

According to the two-step solution illustrated in Fig. 2, the received signal $r(t)$ in (3) is first used to estimate time delays $\delta_{k}, k=0,1, \ldots, N-1$. More specifically, $r(t)$ for $t \in$ $\left[(k-1) T_{\mathrm{s}}, k T_{\mathrm{s}}\right]$ is used for estimating $\delta_{k}$ using a conventional matched-filter approach. For large SNR. $\beta^{2}$, these time delay estimates are modeled, according to Lemma 1 , as

$$
\hat{\delta}_{k}=h_{k}(\boldsymbol{\lambda})+n_{k}
$$

for $k=0,1, \ldots, N-1$, where $n_{1}, \ldots, n_{N-1}$ are independent and identically distributed Gaussian random variables with zero mean and variance $\sigma_{0}^{2}$. Then, the following proposition states the optimality property of the two-step algorithm in Fig. 2.

Proposition 2: For a given set of time delay measurements $\hat{\boldsymbol{\delta}}=\left[\begin{array}{llll}\hat{\delta}_{0} & \hat{\delta}_{1} & \cdots & \hat{\delta}_{N-1}\end{array}\right]$ modeled by (26), the CRLB for the covariance matrix of an unbiased estimate of $\boldsymbol{\lambda}$ is the same as the expression in (16), where the FIM is given by (11)-(13).

Proof: From the model in (26) for the time delay estimates, the distribution of $\hat{\boldsymbol{\delta}}$ for a given $\boldsymbol{\lambda}$ is obtained as

$$
p_{\boldsymbol{\lambda}}(\hat{\boldsymbol{\delta}})=\frac{1}{\left(\sqrt{2 \pi} \sigma_{0}\right)^{N}} \exp \left\{-\frac{1}{2 \sigma_{0}^{2}} \sum_{k=0}^{N-1}\left(\hat{\delta}_{k}-h_{k}(\boldsymbol{\lambda})\right)^{2}\right\} .
$$

Then, the FIM can be shown to be equal to that given by (11) and (12), by using the fact that the expression for $\sigma_{0}^{2}$ in Lemma 1 , $\sigma_{0}^{2}=1 /\left(4 \pi^{2} \beta^{2} \mathrm{SNR}\right)$, can be expressed as $\sigma_{0}^{2}=N \sigma^{2} / \tilde{E}[33]$.
Note that the LS estimator in (23) can be shown to be the ML estimator for $\boldsymbol{\lambda}$ according to the signal model in (26). Since the ML estimator asymptotically achieves the CRLB [29], which is equal to the CRLB given by (11)-(16) according to Proposition 2 , the LS solution in (23) provides an asymptotically optimal solution for estimating $\boldsymbol{\lambda}$ under the conditions stated in Lemma 1.

\section{Multipath Case}

\section{A. General Theory}

For the multipath case, the unknown parameter vector $\theta$ in (5) consists of both the parameters of the displacement function, $\boldsymbol{\lambda}$, and those of the multipath channel, $\boldsymbol{\tau}$ and $\boldsymbol{\alpha}$. Therefore, the FIM in (8) and (9) can be expressed as

$$
\mathbf{I}_{\mathrm{MP}}=\left[\begin{array}{lll}
\mathbf{I}_{\boldsymbol{\lambda}} & \mathbf{I}_{\boldsymbol{\lambda} \tau} & \mathbf{I}_{\boldsymbol{\lambda} \boldsymbol{\alpha}} \\
\mathbf{I}_{\boldsymbol{\lambda} \boldsymbol{\tau}}^{T} & \mathbf{I}_{\boldsymbol{\tau} \boldsymbol{\tau}} & \mathbf{I}_{\boldsymbol{\alpha} \boldsymbol{\alpha}} \\
\mathbf{I}_{\boldsymbol{\lambda} \boldsymbol{\alpha}}^{T} & \mathbf{I}_{\boldsymbol{\tau} \boldsymbol{\alpha}}^{T} & \mathbf{I}_{\boldsymbol{\alpha} \boldsymbol{\alpha}}
\end{array}\right]
$$

where the subscript MP represents the multipath case, and $\mathbf{I}^{T}$ denotes the transpose of $\mathbf{I}$.

The submatrices in (28) can be obtained from (4), (8), and (9) after some manipulation. For example, it can be shown that $\mathbf{I}_{\boldsymbol{\lambda} \boldsymbol{\lambda}}=\alpha_{b}^{2} \mathbf{I}_{\mathrm{SP}}$, where $\mathbf{I}_{\mathrm{SP}}$ is the FIM for the single-path case, given by (11) and (12). The expressions for the remaining submatrices of $\mathbf{I}_{\mathrm{MP}}$ are given in Appendix B.

Direct calculation of the CRLB for frequency estimation, $\left[\mathbf{I}_{\mathrm{MP}}^{-1}\right]_{11}$, requires the inversion of the $(2 L+K) \times(2 L+K)$ FIM in (28), which is computation intensive in most cases. However, it can be shown that the same CRLB can be obtained by inverting a reduced FIM depending on the pulsewidth, the displacement function, and the multipath channel profile. Assume that $\max _{k \in\{0, \ldots, N-1\}}\left|h_{k}(\boldsymbol{\lambda})\right|<T_{\mathrm{w}}$; that is, the maximum time displacement is always smaller than the pulsewidth ${ }^{6}$. Also assume that the interval $T_{\mathrm{p}}$ between consecutive pulses in a burst is sufficiently large so that there is no inter-pulse interference; i.e., $T_{\mathrm{p}}>\tau_{L}-\tau_{1}+2 T_{\mathrm{w}}$. Let $\mathcal{L}_{1}^{(\mathrm{u})}\left(\mathcal{L}_{1}^{(\mathrm{l})}\right)$ denote the set of indices of multipath components that arrive after (before) the $b$ th multipath component and partially overlap with it in at least one burst interval. In other words

$$
\begin{aligned}
\mathcal{L}_{1}^{(\mathrm{u})} & =\left\{l>b \mid \tau_{l}<\max _{k} h_{k}(\boldsymbol{\lambda})+\tau_{b}+T_{\mathrm{w}}\right\} \\
\mathcal{L}_{1}^{(1)} & =\left\{l<b \mid \tau_{l}>\min _{k} h_{k}(\boldsymbol{\lambda})+\tau_{b}-T_{\mathrm{w}}\right\} .
\end{aligned}
$$

Similarly, let $\mathcal{L}_{n}^{(\mathrm{u})}\left(\mathcal{L}_{n}^{(\mathrm{l})}\right)$ represent the set of indices of multipath components that arrive after (before) the multipath component corresponding to the maximum (minimum) element of $\mathcal{L}_{n-1}^{(\mathrm{u})}\left(\mathcal{L}_{n-1}^{(\mathrm{l})}\right)$, and partially overlap with it; i.e.,

$$
\begin{aligned}
\mathcal{L}_{n}^{(\mathrm{u})} & =\left\{l>l_{n-1}^{(\mathrm{u})} \mid \tau_{l}<\tau_{l_{n-1}^{(\mathrm{u})}}+T_{\mathrm{w}}\right\}, \\
\text { for } n & =2, \ldots, N_{L}^{(u)} \\
\mathcal{L}_{n}^{(\mathrm{l})} & =\left\{l<l_{n-1}^{(\mathrm{l})} \mid \tau_{l}>\tau_{l_{n-1}^{(1)}}-T_{\mathrm{w}}\right\}, \\
\text { for } n & =2, \ldots, N_{L}^{(\mathrm{l})}
\end{aligned}
$$

${ }^{6}$ This is a practical assumption for the cases of respiration or heartbeat rate estimation. For example, for a pulsewidth of $0.5 \mathrm{~ns}$, the maximum displacement should be smaller than $15 \mathrm{~cm}$, which is always satisfied for those scenarios. 
where $l_{n-1}^{(\mathrm{u})}=\max \left\{\mathcal{L}_{n-1}^{(\mathrm{u})}\right\}, l_{n-1}^{(\mathrm{l})}=\min \left\{\mathcal{L}_{n-1}^{(1)}\right\}$, and $N_{L}^{(\mathrm{u})}\left(N_{L}^{(\mathrm{l})}\right)$ represents the number of consecutive non-empty sets $\mathcal{L}_{n}^{(\mathrm{u})}\left(\mathcal{L}_{n}^{(\mathrm{l})}\right)$ starting from $\mathcal{L}_{1}^{(\mathrm{u})}\left(\mathcal{L}_{1}^{(\mathrm{l})}\right)$. Note that $N_{L}^{(\mathrm{u})}$ and $N_{L}^{(1)}$ can be expressed as $N_{L}^{(\mathrm{u})}=\min \left\{n \geq 0 \mid \mathcal{L}_{n+1}^{(\mathrm{u})}=\emptyset\right\}$ and $N_{L}^{(1)}=\min \left\{n \geq 0 \mid \mathcal{L}_{n+1}^{(1)}=\emptyset\right\}$, respectively 7 .

From (29)-(32), the set of multipath components that partially overlap with the $b$ th multipath component, directly or indirectly, can be expressed as

$$
\mathcal{L}^{(\mathrm{u})}=\bigcup_{n=1}^{N_{L}^{(\mathrm{u})}} \mathcal{L}_{n}^{(\mathrm{u})} \text { and } \mathcal{L}^{(\mathrm{l})}=\bigcup_{n=1}^{N_{L}^{(1)}} \mathcal{L}_{n}^{(1)}
$$

for the multipath components arriving after and before the $b$ th one, respectively. Note that $\mathcal{L}_{1}^{(\mathrm{u})}$ and $\mathcal{L}_{1}^{(\mathrm{l})}$ in (29) and (30) represent the sets of multipath components that partially overlap with the $b$ th one directly, while $\mathcal{L}_{n}^{(\mathrm{u})}$ and $\mathcal{L}_{n}^{(1)}$ for $n>1$ in (31) and (32) represent the sets of multipath components that partially overlap with the $b$ th one indirectly; i.e., through other multipath components. For example, the multipath components in $\mathcal{L}_{2}^{(\mathrm{u})}$ (or $\mathcal{L}_{2}^{(1)}$ ) do not overlap with the $b$ th one, but partially overlap with at least one multipath component that partially overlaps with the $b$ th one; i.e., they partially overlap with the $b$ th multipath component through some other multipath components.

Then, the set of multipath components that (partially) overlap the $b$ th one directly or indirectly, including the $b$ th multipath component itself, can be expressed as

$$
\tilde{\mathcal{L}}=\mathcal{L}^{(\mathrm{u})} \cup\{b\} \cup \mathcal{L}^{(1)}
$$

where $\mathcal{L}^{(\mathrm{u})}$ and $\mathcal{L}^{(1)}$ are as given in (33).

Then, the following result states that a reduced FIM based on (33) and (34) can be used to obtain the CRLB for estimating the unknown parameters of the displacement function.

Proposition 3: For $\max _{k \in\{0, \ldots, N-1\}}\left|h_{k}(\boldsymbol{\lambda})\right|<T_{\mathrm{w}}$, the CRLB for $\lambda$ calculated from the FIM in (28) is the same as the CRLB that is obtained by using the following reduced FIM:

$$
\tilde{\mathbf{I}}_{\mathrm{MP}}=\left[\begin{array}{lll}
\mathbf{I}_{\lambda \boldsymbol{\lambda}} & \mathbf{I}_{\boldsymbol{\lambda} \tilde{\boldsymbol{\tau}}} & \mathbf{I}_{\boldsymbol{\lambda} \tilde{\boldsymbol{\alpha}}} \\
\mathbf{I}_{\boldsymbol{\lambda} \tilde{\tau}}^{T} & \mathbf{I}_{\tilde{\tau} \tilde{\boldsymbol{\tau}}} & \mathbf{I}_{\tilde{\tau} \tilde{\boldsymbol{\alpha}}} \\
\mathbf{I}_{\boldsymbol{\lambda} \tilde{\boldsymbol{\alpha}}}^{T} & \mathbf{I}_{\tilde{\tau} \tilde{\boldsymbol{\alpha}}}^{T} & \mathbf{I}_{\tilde{\alpha} \tilde{\boldsymbol{\alpha}}}
\end{array}\right]
$$

where 8

$$
\begin{aligned}
& \mathbf{I}_{\boldsymbol{\lambda} \tilde{\boldsymbol{\tau}}}=\left[\mathbf{I}_{\boldsymbol{\lambda} \boldsymbol{\tau}}\right]_{: \tilde{\mathcal{L}}}, \quad \mathbf{I}_{\boldsymbol{\lambda} \tilde{\boldsymbol{\alpha}}}=\left[\mathbf{I}_{\boldsymbol{\lambda} \boldsymbol{\alpha}}\right]_{: \tilde{\mathcal{L}}} \\
& \mathbf{I}_{\tilde{\boldsymbol{\tau}} \tilde{\boldsymbol{\tau}}}=\left[\mathbf{I}_{\boldsymbol{\tau} \boldsymbol{\tau}}\right]_{\tilde{\mathcal{L}} \tilde{\mathcal{L}}}, \quad \mathbf{I}_{\tilde{\boldsymbol{\alpha}} \tilde{\boldsymbol{\alpha}}}=\left[\mathbf{I}_{\boldsymbol{\alpha} \boldsymbol{\alpha}}\right]_{\tilde{\mathcal{L}} \tilde{\mathcal{L}}}, \quad \mathbf{I}_{\tilde{\tau} \tilde{\boldsymbol{\alpha}}}=\left[\mathbf{I}_{\boldsymbol{\tau} \boldsymbol{\alpha}}\right]_{\tilde{\mathcal{L}} \tilde{\mathcal{L}}}
\end{aligned}
$$

with $\tilde{\mathcal{L}}$ being given by (29)-(34).

Proof: See Appendix C.

Proposition 3 states that inversion of the reduced FIM in (35) is sufficient to obtain the CRLB for estimating the parameters

\footnotetext{
${ }^{7}$ Note that (31) and (32) are defined only for $N_{L}^{(\mathrm{u})}>1$ and $N_{L}^{(1)}>1$, respectively.

${ }^{8}[\mathbf{I}]_{: \mathcal{A}}$ represents a matrix with its $j$ th column being equal to the $\mathcal{A}(j)$ th column of $\mathbf{I}$ for $j=1, \ldots,|\mathcal{A}|$, where $\mathcal{A}(j)$ is the $j$ th element of set $\mathcal{A}$ and $|\mathcal{A}|$ represents the number of elements in $\mathcal{A}$. Similarly, $[\mathbf{I}]_{\mathcal{A}_{1} \mathcal{A}_{2}}$ represents a $\left|\mathcal{A}_{1}\right| \times\left|\mathcal{A}_{2}\right|$ matrix whose element in the $k$ th row of the $j$ th column is equal to the element of $\mathbf{I}$ in the $\mathcal{A}_{1}(k)$ th row of the $\mathcal{A}_{2}(j)$ th column.
}

of the displacement function, $\boldsymbol{\lambda}$. The reduced FIM is a $(K+$ $\left.2 N_{\tilde{L}}\right) \times\left(K+2 N_{\tilde{L}}\right)$ matrix, where $N_{\tilde{L}}$ represents the number of elements in $\tilde{\mathcal{L}}$, which is given by (34). The amount of complexity reduction depends on $N_{\tilde{L}}$, which is a random variable characterized by the channel statistics, the pulsewidth and the displacement function, as can be observed from (29)-(33).

For a multipath channel model with Poisson arrivals for the multipath components [34], it is possible to obtain the (approximate) statistics for $N_{\tilde{L}}$. Note that indoor UWB channel models [35], [36] also specify Poisson arrivals for clusters and multipath components inside each cluster9.

Let $C_{i}^{(\mathrm{u})}$, for $i=1, \ldots, L-b$, denote the event that the $(b+i)$ th multipath component partially overlaps with the $(b+$ $i-1)$ th multipath component, or it partially overlaps with the $b$ th one without partially overlapping with the $(b+i-1)$ th one. Similarly, $C_{i}^{(1)}$, for $i=1, \ldots, b-1$, represents the event that the $(b-i)$ th multipath component partially overlaps with the $(b-i+1)$ th multipath component, or it partially overlaps with the $b$ th one without partially overlapping with the $(b-i+1)$ th one. Also let $Y^{(\mathrm{u})}$ and $Y^{(\mathrm{l})}$ represent, respectively, the number of elements in $\mathcal{L}^{(\mathrm{u})}$ and $\mathcal{L}^{(\mathrm{l})}$ in (33). Then, the probability mass function $(\mathrm{PMF})$ of $Y^{(\mathrm{u})}$ can be expressed as

$$
\begin{aligned}
P\left(Y^{(\mathrm{u})}=n\right)= & P\left(C_{1}^{(\mathrm{u})}\right) \prod_{j=2}^{n} P\left(C_{j}^{(\mathrm{u})} \mid C_{j-1}^{(\mathrm{u})} \cdots C_{1}^{(\mathrm{u})}\right) \\
& \times\left(1-P\left(C_{n+1}^{(\mathrm{u})} \mid C_{n}^{(\mathrm{u})} \cdots C_{1}^{(\mathrm{u})}\right)\right)
\end{aligned}
$$

for $n=1, \ldots, L-b$, and $P\left(Y^{(\mathrm{u})}=0\right)=1-P\left(C_{1}^{(\mathrm{u})}\right)$. Note that the PMF in (38) can be obtained by the observation that $Y^{(\mathrm{u})}=n$ implies that each multipath components in the set $\{(b+1), \ldots,(b+n)\}$ either collides with the previous multipath component, or collides with the $b$ th one directly without colliding with the previous one ${ }^{10}$, and that the $(b+n+1)$ th multipath component collides with neither the $(b+n)$ th nor the $b$ th multipath component. A similar expression can be obtained for the PMF of $Y^{(1)}$, as well.

Assume that $h_{M}=\max _{k}\left\{h_{k}(\boldsymbol{\theta})\right\}=-\min _{k}\left\{h_{k}(\boldsymbol{\theta})\right\}, b \gg$ 1 , and $L \gg 1$. For a multipath channel with Poisson arrivals and a single cluster, it can be shown that the events $C_{i}^{(\mathrm{u})}$ for $i=1, \ldots, L-b$ are approximately independent for $h_{M} \ll T_{\mathrm{w}}$ (see Appendix D). Then, the PMF of $Y^{(\mathrm{u})}$ can be obtained from (38), after some manipulation, as seen in (39), shown at the bottom of the page, where $\varphi$ is the rate of the Poisson process. This expression can also be used for $P\left(Y^{(1)}=n\right)$ by replacing $L-b$ with $b-1$. From (39), the expected value of $Y^{(\mathrm{u})}$ can be obtained as

$$
\begin{aligned}
E\left\{Y^{(\mathrm{u})}\right\} \approx\left(e^{\varphi T_{\mathrm{w}}}-e^{\varphi h_{M}}\right) & \left(1-\left(1-e^{-\varphi T_{\mathrm{w}}}\right)^{L-b}\right. \\
\times & \left.\left(1-(L-b+2) e^{-\varphi T_{\mathrm{w}}}\right)\right)
\end{aligned}
$$

${ }^{9}$ To be precise, the IEEE 802.15.4a channel model specifies a mixture of two Poisson processes for the ray arrivals in each cluster [36]. The analysis can be extended to cover that case as well. However, a single Poisson process is considered for clarity of the expressions.

${ }^{10}$ This is possible due to the variable delay of the $b$ th multipath component. 


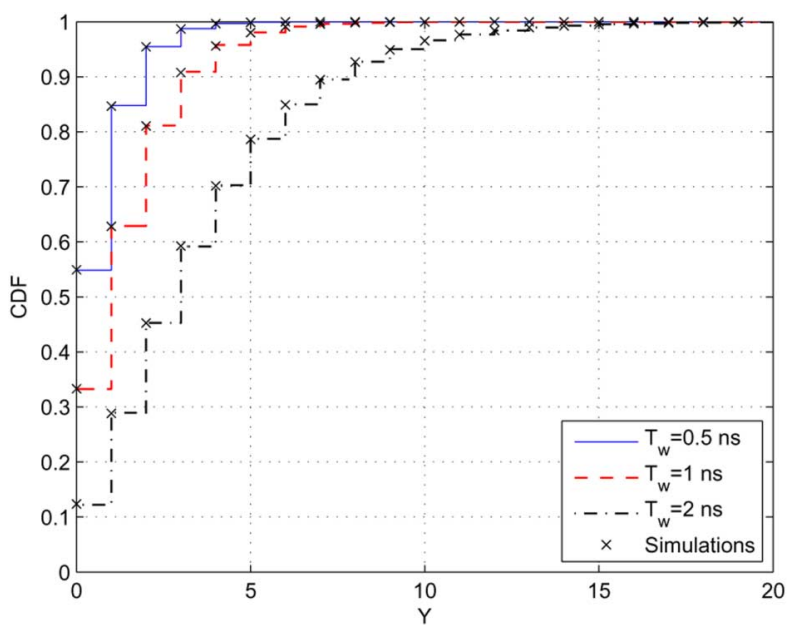

Fig. 3. CDF of $Y$ for various pulsewidths. The lines are obtained from (41), and the cross signs $(x)$ represent the simulation results.

and $E\left\{Y^{(1)}\right\}$ can be obtained from (40) by replacing $(L-b) \mathrm{s}$ by $(b-1)$ s. Then, the expectation of $N_{\tilde{L}}$, the size of $\hat{\mathcal{L}}$ in (34), can be obtained from $E\left\{N_{\tilde{L}}\right\}=E\left\{Y^{(\mathrm{u})}\right\}+E\left\{Y^{(\mathrm{l})}\right\}+1$.

Let $Y=Y^{(\mathrm{u})}+Y^{(\mathrm{l})}$. Due to the independence of $Y^{(\mathrm{u})}$ and $Y^{(1)}$, which is a property of Poisson arrivals, the PMF of $Y$ can be obtained by the convolution of $P\left(Y^{(\mathrm{u})}=n\right)$ in (39) and $P\left(Y^{(1)}=n\right)$ as $P(Y=0)=e^{-2 \varphi\left(T_{\mathrm{w}}+h_{M}\right)}$, and

$$
\begin{aligned}
P(Y=n) \approx & e^{-2 \varphi T_{\mathrm{w}}}\left(1-e^{-\varphi\left(T_{\mathrm{w}}+h_{M}\right)}\right) \\
& \times\left(1-e^{-\varphi T_{\mathrm{w}}}\right)^{n-2} \\
& \times\left(2 e^{-\varphi h_{M}}\left(1-e^{-\varphi T_{\mathrm{w}}}\right)\right. \\
& \left.\quad+\left(1-e^{-\varphi\left(T_{\mathrm{w}}+h_{M}\right)}\right) \Xi(n, b, L)\right)
\end{aligned}
$$

for $n=1, \ldots, L-1$, where

$$
\Xi(n, b, L)= \begin{cases}n-1, & 1 \leq n \leq b \\ b-1, & b<n \leq L-b+1 \\ L-n, & L-b+1<n \leq L-1 \\ 0, & \text { otherwise }\end{cases}
$$

under the assumption that $L>2 b-1 .^{11}$

As an example, the cumulative distribution function (CDF) of $Y$ is plotted in Fig. 3 for various pulsewidths, where $b=20$, $L=100, \varphi=0.5 \mathrm{~ns}^{-1}$, and $h_{M}=0.1 \mathrm{~ns}$ are used. It is observed from the figure that $N_{\tilde{L}}(=Y+1)$ is smaller than 15

\footnotetext{
${ }^{11}$ The expression for $L \leq 2 b-1$ can be obtained in a similar manner.
}

most of the time, which is a considerable complexity reduction compared to 100 multipaths.

\section{B. Resolvable Case}

In the resolvable case, the $b$ th multipath component does not overlap with any other multipath components in any of the bursts ${ }^{12}$. In this case, a simple CRLB expression can be obtained.

Corollary 1: For $N_{L}^{(\mathrm{u})}=N_{L}^{(1)}=0$, the CRLB for $f$ can be expressed as

$$
\operatorname{Var}\{\hat{f}\} \geq \frac{\left[\mathbf{I}_{\mathrm{SP}}^{-1}\right]_{11}}{\alpha_{b}^{2}}+\frac{\sigma^{2}}{\alpha_{b}^{2} \tilde{E}_{b}} \frac{\left(\left[\boldsymbol{\Upsilon}^{-1}\right]_{1:} \boldsymbol{\gamma}\right)^{2}}{\left(1-\frac{1}{N} \boldsymbol{\gamma}^{T} \boldsymbol{\Upsilon}^{-1} \boldsymbol{\gamma}\right)}
$$

where $\mathbf{I}_{\mathrm{SP}}$ is the FIM for the single path case in Section IV

$$
[\boldsymbol{\Upsilon}]_{i j}= \begin{cases}\sum_{k=0}^{N-1}\left(\frac{\partial h_{k}(\boldsymbol{\lambda})}{\partial \lambda_{i}}\right)^{2}, & i=j, \\ \sum_{k=0}^{N-1} \frac{\partial h_{k}(\boldsymbol{\lambda})}{\partial \lambda_{i}} \frac{\partial h_{k}(\boldsymbol{\lambda})}{\partial \lambda_{j}}, & i \neq j\end{cases}
$$

and $\gamma=\left[\gamma_{1} \cdots \gamma_{K}\right]^{T}$, with

$$
\gamma_{i}=\sum_{k=0}^{N-1} \frac{\partial h_{k}(\boldsymbol{\lambda})}{\partial \lambda_{i}}
$$

Proof: See Appendix E.

It is observed from Corollary 1 that the CRLB does not depend on the nominal delay of the multipath component reflecting from the object, $\tau_{b}$. In other words, the exact pulse position is not crucial in the absence of partial overlaps with the $b$ th component. Also, it is noted from (11), (12) and (43) that the CRLB is inversely proportional to $\alpha_{b}^{2} \tilde{E}_{b} / \sigma^{2}$.

For a sinusoidal displacement function as in (18), a simple approximate result can be obtained for a large number of bursts, $N$.

Proposition 4: Consider a sinusoidal displacement function given by $h_{k}(\boldsymbol{\lambda})=A \sin \left(2 \pi f k T_{\mathrm{s}}+\phi\right)$. Assume that $T_{\mathrm{s}}<$ $1 /(4 f)$ and $N_{L}^{(\mathrm{u})}=N_{L}^{(1)}=0$. Then, as $N \longrightarrow \infty$, the CRLB for $f$ multiplied by $N^{2}$ is expressed as

$$
\lim _{N \rightarrow \infty} N^{2}\left[\mathbf{I}_{\mathrm{MP}}^{-1}\right]_{11}=\frac{6 \sigma^{2}}{\pi^{2} \tilde{E}_{b} \alpha_{b}^{2} A^{2} T_{\mathrm{s}}^{2}} .
$$

Proof: See Appendix F.

${ }^{12}$ Multipath components other than the $b$ th one can still overlap with each other.

$$
P\left(Y^{(\mathrm{u})}=n\right) \approx \begin{cases}e^{-\varphi\left(T_{\mathrm{w}}+h_{M}\right)}, & n=0 \\ e^{-\varphi T_{\mathrm{w}}}\left(1-e^{-\varphi\left(T_{\mathrm{w}}+h_{M}\right)}\right)\left(1-e^{-\varphi T_{\mathrm{w}}}\right)^{n-1}, & n=1, \ldots, L-b \\ 0, & \text { otherwise }\end{cases}
$$


For large $N$, the CRLB for $f$ can be approximated, from (46), as

$$
\left[\mathbf{I}_{\mathrm{MP}}^{-1}\right]_{11} \approx \frac{6 \sigma^{2}}{\pi^{2} \tilde{E}_{b} \alpha_{b}^{2} A^{2} T^{2}} \approx \frac{0.0154}{\mathrm{SNR}_{b} \beta^{2} A^{2} T^{2}}
$$

where the second equation is obtained from (15) with $\mathrm{SNR}_{b}=$ $\alpha_{b}^{2} E_{b} / \sigma^{2}$. Similar to the single-path case in Section IV, it is observed from (47) that as the maximum time displacement amount $A$ or the observation interval $T$ increases, the minimum achievable variance decreases with the square of these terms. In addition, $\alpha_{b}^{2} \tilde{E}_{b} / \sigma^{2}$ (or, $\mathrm{SNR}_{b} \beta^{2}$ ) is inversely proportional to the CRLB. It is also important to note from (47) that the approximate expression for the minimum achievable accuracy is independent of the true frequency and phase values.

\section{Suboptimal Solutions}

The ML solution in Section III requires correlation of the incoming signal with a template signal for all different values of the parameter $\boldsymbol{\theta}$ as described in (7), which has prohibitive complexity for practical applications. In order to have an asymptotically optimal solution based on matched filter (correlation) processing at individual burst intervals and LS estimation, as in Section IV-C, the matched filter template for each burst interval should in general match to the received signal in a burst, which consists of $L$ multipath components. In that case, the time delay estimates for each multipath component can be approximated by Gaussian random variables around the true time delays [31]. Hence, an LS estimator based on matched filter outputs from different burst intervals can be designed. However, the complexity of the overall algorithm is still very high, since the matched filtering at each burst interval needs to be performed over unknown parameters of the multipath channel.

The only special case in which the two-step solution based on matched filtering and LS estimation results in a low-complexity and asymptotically optimal solution is the resolvable case; i.e., the $b$ th multipath component does not collide with any other multipath components in any burst interval. In this case, if the nominal delay of the $b$ th multipath component, $\tau_{b}$, is known (for example, by assuming the knowledge of the distance between the object, e.g., a human chest, and the transceiver), matched filtering with a template signal matched to the received pulse [ $w_{b}(t)$ in (4)] yields an estimate for $h_{k}(\boldsymbol{\theta})$ for each $k(k=$ $0, \ldots, N-1)$, which has Gaussian distribution around the true displacement value, as in Section IV-C, for sufficiently large SNR and/or effective bandwidth. In other words, the resolvable case with a known $\tau_{b}$ is the same as the single-path case in Section IV-C, since the other multipath components has no effect on the $b$ th one. Therefore, the analysis in Section IV-C also proves that the two-step approach can asymptotically attain the CRLB for $\boldsymbol{\lambda}$; i.e., $\mathbf{I}_{\boldsymbol{\lambda} \boldsymbol{\lambda}}$, for the resolvable case.

In practice, delays between multipath components in indoor UWB channels can be smaller than the pulsewidth. Also there is no prior information about the collision of the multipath component from the object, the $b$ th multipath component, with any other multipath components. Therefore, it may not be very practical to assume a resolvable case and employ matched filtering based on a single pulse. In order to overcome the effects of multipath components interfering with the $b$ th component, a filtering approach can be applied. Since the interfering multipath components are all stationary whereas the signal component due to the object is time varying, a high pass filter can be applied to the received signal to eliminate the signal components that do not vary among consecutive burst intervals ${ }^{13}$. The easiest way to implement such a high pass filter is to subtract the average of the received signals in different bursts from each signal in a given burst, as considered in [23]. After such a filtering operation, the filtered signal can be obtained from (3) and (4) as

$$
\begin{aligned}
\bar{r}(t)= & \frac{\alpha_{b}}{\sqrt{N}} \sum_{k=0}^{N-1}\left(w_{b}\left(t-k T_{\mathrm{s}}-\tau_{b}-h_{k}(\boldsymbol{\lambda})\right)\right. \\
& \left.-\frac{1}{N} \sum_{m=0}^{N-1} w_{b}\left(t-k T_{\mathrm{s}}-\tau_{b}-h_{m}(\boldsymbol{\lambda})\right)\right)+\bar{n}(t)
\end{aligned}
$$

where $\bar{n}(t)$ represents the filtered noise. After the filtering, the Fourier transform (FT) of the filtered signal $\bar{r}(t)$ along a certain set of samples yields an estimate for the frequency parameter of the displacement function [23]. Due to the practicality of this filtering approach for multipath channels, the performance analysis in Section VI will focus on the study of this approach instead of the matched filter-based schemes.

\section{Simulation Results}

In this section, numerical studies and simulations are performed to evaluate the exact and approximate CRLB expressions, and to investigate the performance of suboptimal algorithms discussed in Sections IV-C and V-C.

A sinusoidal displacement function, as in (18), is considered, and the following Gaussian mono-cycle is employed as the received pulse shape for all multipath components [39]:

$$
p_{\mathrm{rx}}(t)=\left(1-\frac{4 \pi t^{2}}{\zeta^{2}}\right) \frac{e^{-\left(2 \pi t^{2} / \zeta^{2}\right)}}{\sqrt{E_{p}}}
$$

where $E_{p}$ is used to adjust the energy of the pulse in the simulations, and $\zeta$ determines the pulsewidth $\left(T_{\mathrm{w}} \approx 2.5 \zeta\right)$.

In Fig. 4, the exact and approximate CRLB expressions in (19) and (22), respectively, are compared for various SNRs and pulsewidths ${ }^{14}$. The system parameters are $T_{\mathrm{s}}=0.1 \mathrm{~s}, f=$ $1.1 \mathrm{~Hz}, A=0.1 \mathrm{~ns}, \phi=0$, and $N=50$. Although the frequency and phase are set to certain values, the results are practically the same for any frequency satisfying $f<2.5 \mathrm{~Hz}$ and any phase. From Fig. 4, it is observed that the exact and approximate CRLBs match very closely for all SNR and $T_{\mathrm{w}}$ values. In addition, the accuracy increases as the pulsewidth is decreased. This is intuitive since higher time resolution results in better localization of the object. This can be also observed from (22), since shorter pulses have larger $\tilde{E}$ as can be deduced from (15). In Fig. 5, the lower bounds for the exact and approximate CRLB

\footnotetext{
${ }^{13}$ In radar terminology, the unwanted (interfering) multipath components are called clutter. Various filtering approaches were studied in the radar literature in order to mitigate the effects of clutter for the case in which the object of interest ("target") is moving while the objects generating the clutter are stationary [28], [37], [38]. The conventional clutter removal approach in such a case is to use high-pass filters. [37].

${ }^{14}$ Square-roots of the expressions are plotted and the lower bounds are obtained in units of $\mathrm{Hz}$.
} 


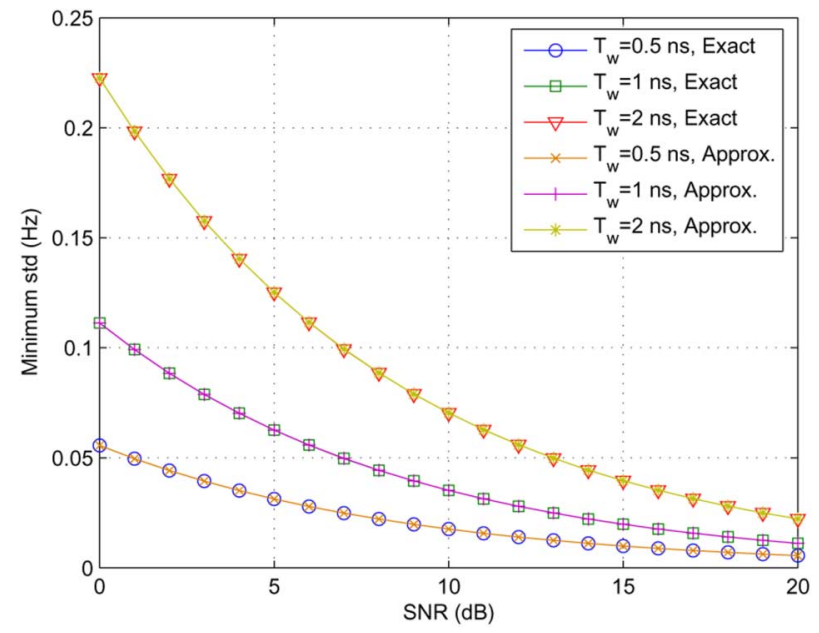

Fig. 4. Exact and approximate CRLB versus SNR for various pulsewidths, where $N=50$ bursts are transmitted.

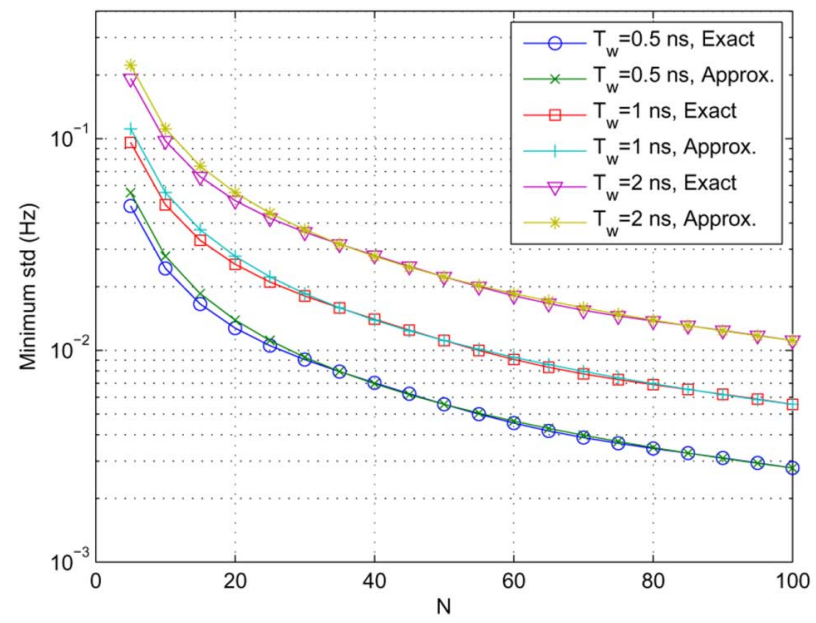

Fig. 5. Exact and approximate CRLB versus the number of bursts, $N$, for various pulsewidths at $\mathrm{SNR}=20 \mathrm{~dB}$.

expressions are plotted against the number of bursts, $N$, for various pulsewidths, at SNR $=20 \mathrm{~dB}$. It is observed from the figure that the approximation gets very accurate when more than 30 bursts are transmitted.

In Fig. 6, all the parameters are the same as in the previous case, except that 700 pulse bursts are employed as in the experiment in [23]. The lower bounds are plotted versus pulsewidth for various SNRs. It is observed again that as the pulsewidth decreases, the accuracy of the estimation increases. Moreover, the slope decreases as the SNR increases, which means that for high SNRs, the loss in accuracy when using wider pulses (pulses with smaller bandwidths) is smaller than the loss for low SNRs.

Fig. 7 compares the performance of the suboptimal LS solution in Section IV-C with the exact and approximate lower bounds for $N=50, T_{\mathrm{w}}=1 \mathrm{~ns}, T_{\mathrm{s}}=0.1 \mathrm{~s}$, and $A=0.1 \mathrm{~ns}$. For the LS solution, averages over 10,000 realizations are averaged to calculate root-mean-square errors (RMSEs) for each SNR. For each realization, random noise, $f$ and $\phi$ values are generated. Considering a respiration rate estimation scenario, $f$ is generated randomly from $[0.5,1.5] \mathrm{Hz}$. From Fig. 7, it is observed that for large SNR, the performance of the LS solution converges to the lower bound, as expected.

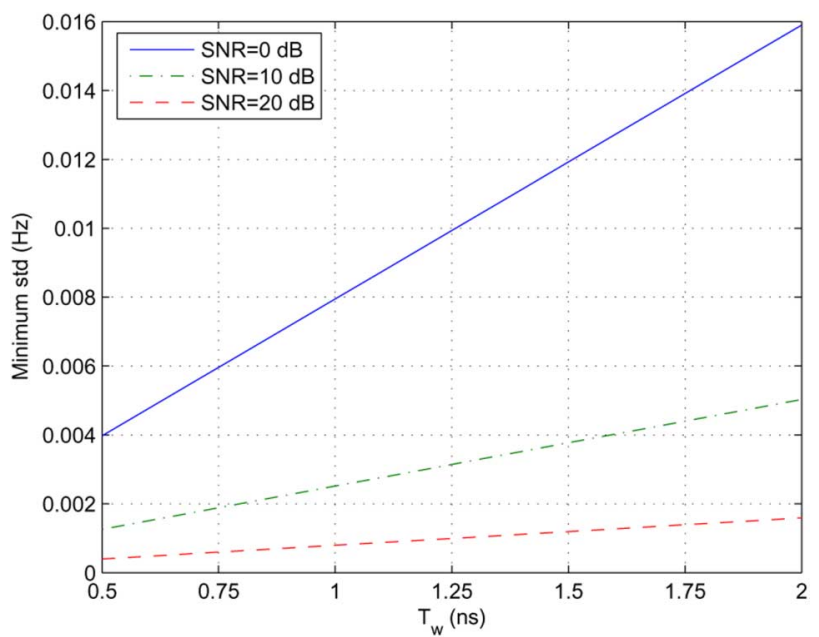

Fig. 6. CRLB versus pulsewidth for various SNRs, where $N=700$ bursts are transmitted.

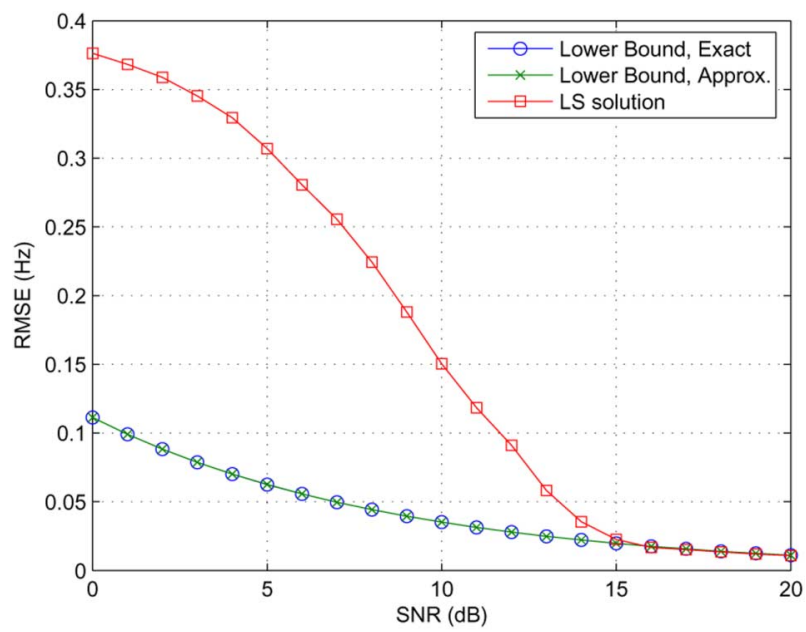

Fig. 7. RMSE versus SNR for the LS solution and the exact and approximate CRLBs for $N=50$ bursts.

In Fig. 8, a resolvable multipath channel as described in Section V-B is considered, and exact and approximate CRLBs are plotted against the number of bursts, $N$, for various pulsewidths. The received multipath component has an amplitude of 0.05 ; i.e., $\left|\alpha_{b}\right|=0.05$, the SNR is $30 \mathrm{~dB}$, and the other system parameters are as in the previous case. Both the generic expression in Proposition 3, and the simplified expression in Corollary 1 are plotted in order to verify the simplified CRLB expression given by (43)-(45). It is observed from the figure that the approximate expression given by (46) gets very close to the exact CRLB after around $N=25$. Also, the CRLBs get smaller as the pulsewidth decreases, since the narrower pulses provide more resolution.

In Fig. 9, the channel models for line-of-sight residential (CM-1) and office (CM-3) environments according to the IEEE 802.15.4a channel model [36] are considered ${ }^{15}$, and the average CRLB expressions, averaged over 100 channel realizations, are plotted. The system parameters are given by $A=0.1 \mathrm{~ns}$, $f=1.1 \mathrm{~Hz}, \phi=0, T_{\mathrm{s}}=0.1 \mathrm{~s}, N=50, T_{\mathrm{w}}=1 \mathrm{~ns}$, and the

\footnotetext{
${ }^{15}$ Note that the IEEE 802.15 .4a channel models may not be very suitable for the scenario considered in this paper. However, the main purpose is to verify the theoretical analysis.
} 


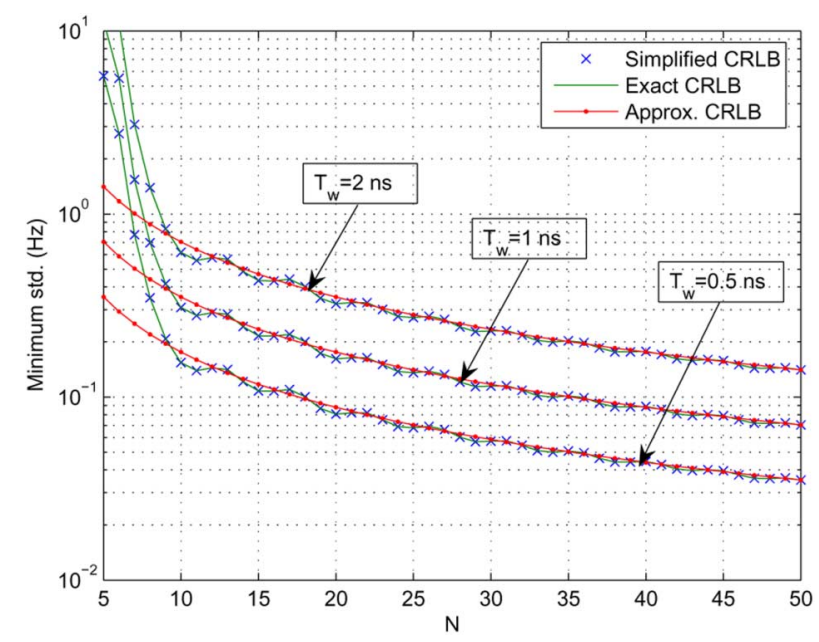

Fig. 8. Exact and approximate CRLB versus $N$ for various pulsewidths, at $\mathrm{SNR}=30 \mathrm{~dB}$.

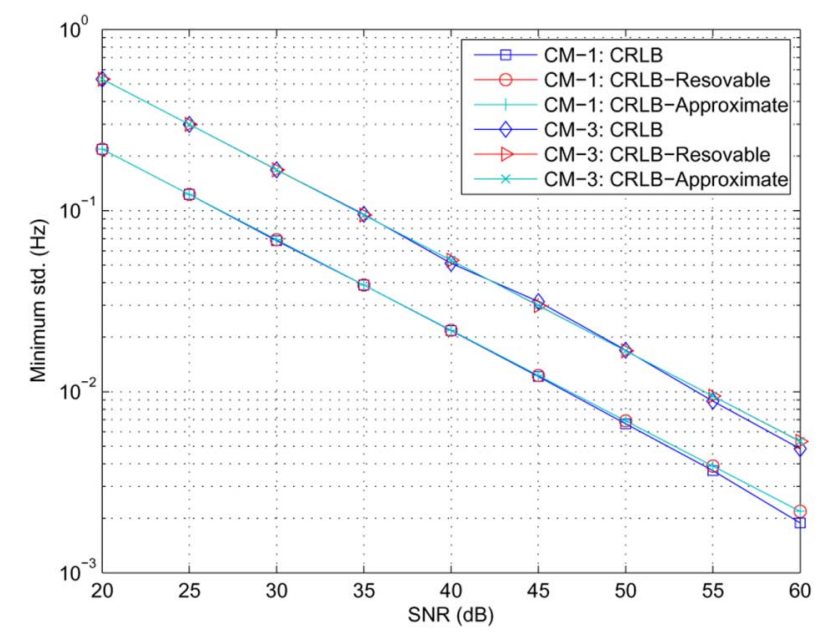

Fig. 9. CRLB versus SNR for CM-1 and CM-3 of the IEEE 802.15.4a channel model [36], where $N=50$ bursts are transmitted.

nominal round-trip time of the multipath component reflecting from the object (with respect to the first multipath component arriving at the receiver) is assumed to be $20 \mathrm{~ns}$. Then, at each channel realization, the multipath component closest to the delay of $20 \mathrm{~ns}$ is considered as the one reflecting from the object. It is noted from the figure that both the generic expression in Proposition 3 and the exact and approximate expressions for the resolvable case, (43)-(45) and (47), respectively, yield very close CRLBs. Moreover, the lower bounds for CM- 1 are smaller than the ones for $\mathrm{CM}-3$, since the average power of the multipath component closest to the 20 ns delay is $4.4 \mathrm{~dB}$ larger for CM-1 than that for CM-3. Also note that for reliable estimation in multipath environments, an SNR of around $40 \mathrm{~dB}$ may be needed, which is not a very large value considering the fact that each burst can be transmitted in a time interval on the order of seconds $(0.1 \mathrm{~s} \text {. in the simulations })^{16}$.

\footnotetext{
${ }^{16}$ Since the observation time is on the order of seconds, considerable signal energy can be collected at the receiver and quite large SNR values can be observed depending, among other factors, on the distance from the object, the propagation environment, and the type of the object.
}

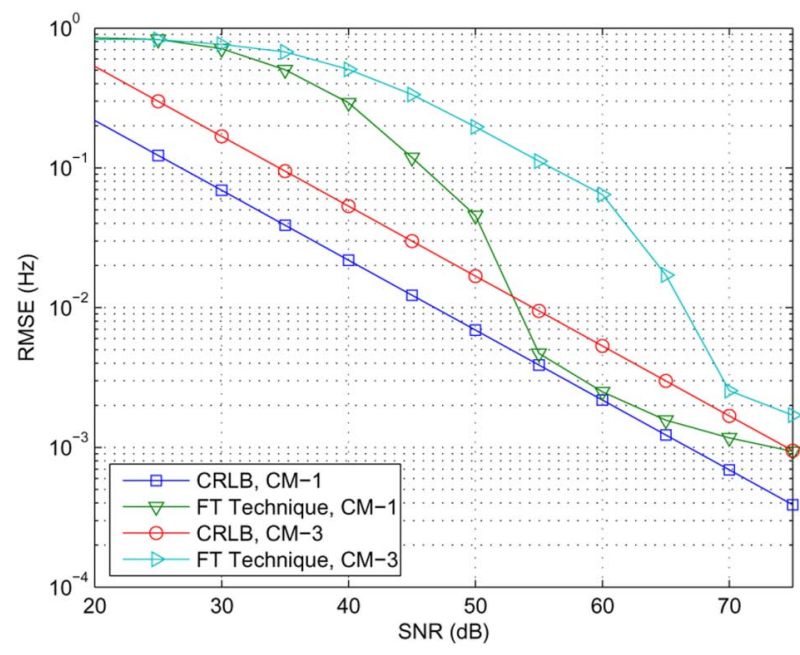

Fig. 10. RMSE of the FT-based solution [23] and CRLB versus SNR for $N=$ 50 bursts. Averaging over 100 realizations of CM-1 and CM-3 are performed.

Finally, Fig. 10 compares the performance of the FT-based algorithm [23] described in Section $\mathrm{V}-\mathrm{C}$ with the average CRLBs for CM-1 and CM-3. The same parameters as for Fig. 9 are used, except that the frequency $f$ is generated randomly from $[0.5,1.5] \mathrm{Hz}$. For the FT-based scheme, a sampling rate of $40 \mathrm{GHz}$ is assumed [23]. It is observed from Fig. 10 that the FT-based scheme gets quite close to the lower bound for high SNR values ${ }^{17}$. However, for very high SNRs, the sampling errors cause an error floor.

\section{CONCLUSION}

Theoretical limits for estimation of signal parameters related to a periodically moving object has been studied for pulse-based UWB systems. First, a generic expression for the CRLB has been obtained for estimating the parameters of a moving object. Then, the single-path scenario has been studied, and exact and approximate closed-form CRLB expressions have been derived. Also, a two-step asymptotically optimal algorithm has been proposed. Then, the analysis has been extended to the multipath case, and CRLBs and suboptimal algorithms have been investigated. Simulations results have been used to verify the analysis and to evaluate the theoretical limits for a practical respiration rate estimation scenario.

\section{APPENDIX}

A. Proof of Proposition 1: Consider the following relation [40]:

$$
\begin{aligned}
\sum_{k=0}^{N-1} \sin (k y+x)=\sin (x+ & \left.\frac{N-1}{2} y\right) \\
& \times \sin \left(\frac{N y}{2}\right) \operatorname{cosec}\left(\frac{y}{2}\right)
\end{aligned}
$$

with $x=2 \phi$ and $y=4 \pi f T_{\mathrm{s}}$.

From (50) and its derivatives with respect to $x$ and/or $y$, closed-form expressions for $X_{11}, X_{22}, X_{33}, X_{12} X_{13}$, and

${ }^{17}$ The nominal delay of the $b$ th multipath component is assumed to be known in the simulations. 
$X_{23}$ in (20) can be obtained ${ }^{18}$. Then, the limit of $N^{2}\left[\mathbf{I}_{\boldsymbol{\theta}}^{-1}\right]_{11}$ as $N \longrightarrow \infty$ can be calculated, after some manipulation, as in Proposition 1.

In the derivations, $\sin (y / 2)$ is assumed to be nonzero. Hence, the case in which $4 \pi f T_{\mathrm{s}} / 2<\pi / 2$ is considered; i.e., $T_{\mathrm{s}}<$ $1 /(4 f)$, as stated in the proposition.

B. FIM for the Multipath Case: By differentiating (4) with respect to various parameters, the submatrices in (28) can be obtained from (8) and (9). Namely, $\mathbf{I}_{\boldsymbol{\tau} \tau}$ is as seen in (51), shown at the bottom of the page, for $l_{1}, l_{2} \in \mathcal{L}$, where $\tilde{E}_{l}$ is the energy of $w_{l}^{\prime}(t)$ and $\tilde{R}_{m n}(x)=\int_{-\infty}^{\infty} w_{m}^{\prime}(t) w_{n}^{\prime}(t+x) d t$. Similarly, $\mathbf{I}_{\boldsymbol{\alpha} \boldsymbol{\alpha}}$ can be expressed as in (52), shown at the bottom of the page, for $l_{1}, l_{2} \in \mathcal{L}$, where $E_{l}$ is the energy of $w_{l}(t)$ and $R_{m n}(x)=$ $\int_{-\infty}^{\infty} w_{m}(t) w_{n}(t+x) d t$. Also, $\mathbf{I}_{\boldsymbol{\lambda} \boldsymbol{\tau}}$ is given by

$$
\left[\mathbf{I}_{\boldsymbol{\lambda} \tau}\right]_{n l}= \begin{cases}\frac{\alpha_{b}^{2} \tilde{E}_{b}}{N \sigma^{2}} \sum_{k=0}^{N-1} \frac{\partial h_{k}(\boldsymbol{\lambda})}{\partial \lambda_{n}}, & l=b \\ \frac{\alpha_{b} \alpha_{l}}{N \sigma^{2}} \sum_{k=0}^{N-1} \tilde{R}_{b l}\left(\tau_{b}+h_{k}(\boldsymbol{\lambda})-\tau_{l}\right) \frac{\partial h_{k}(\boldsymbol{\lambda})}{\partial \lambda_{n}}, & l \neq b\end{cases}
$$

for $n=1, \ldots, K$ and $l \in \mathcal{L}$, and

$$
\left[\mathbf{I}_{\boldsymbol{\lambda} \boldsymbol{\alpha}}\right]_{n l}= \begin{cases}-\frac{\alpha_{b} \hat{E}_{b}}{N \sigma^{2}} \sum_{k=0}^{N-1} \frac{\partial h_{k}(\boldsymbol{\lambda})}{\partial \lambda_{n}}, & l=b \\ -\frac{\alpha_{b}}{N \sigma^{2}} \sum_{k=0}^{N-1} \hat{R}_{b l}\left(\tau_{b}+h_{k}(\boldsymbol{\lambda})-\tau_{l}\right) \frac{\partial h_{k}(\boldsymbol{\lambda})}{\partial \lambda_{n}}, & l \neq b\end{cases}
$$

${ }^{18}$ The detailed expressions and the derivations are omitted due to space limitations. for $n=1, \ldots, K$ and $l \in \mathcal{L}$, where $\hat{R}_{m n}(x)=\int_{-\infty}^{\infty} w_{m}^{\prime}$ $(t) w_{n}(t+x) d t$ and $\hat{E}_{l}=\hat{R}_{l l}(0)$. Finally, we have (55), shown at the bottom of the page, for $l_{1}, l_{2} \in \mathcal{L}$.

C. Proof of Proposition 3: Let $\tilde{\boldsymbol{\alpha}}$ and $\tilde{\boldsymbol{\tau}}$ represent, respectively, the channel coefficients and the delays of the multipath components that belong to $\tilde{\mathcal{L}}$ in (34). In other words, $\tilde{\boldsymbol{\alpha}}=[\boldsymbol{\alpha}]_{\tilde{\mathcal{L}}}$ and $\tilde{\boldsymbol{\tau}}=[\boldsymbol{\tau}]_{\tilde{\mathcal{L}}}$. Let $\hat{\boldsymbol{\alpha}}$ and $\hat{\boldsymbol{\tau}}$ represent the remaining elements of $\boldsymbol{\alpha}$ and $\boldsymbol{\tau}$, respectively; i.e., $\hat{\boldsymbol{\alpha}}=[\boldsymbol{\alpha}]_{\mathcal{L}-\tilde{\mathcal{L}}}$ and $\hat{\boldsymbol{\tau}}=[\boldsymbol{\tau}]_{\mathcal{L}-\tilde{\mathcal{L}}}$.

Instead of calculating the FIM as in (28), one can consider the FIM $\check{\mathbf{I}}$ for the rearranged parameter vector $\tilde{\boldsymbol{\theta}}=\left[\begin{array}{lllll}\lambda & \tilde{\boldsymbol{\tau}} & \tilde{\boldsymbol{\alpha}} & \hat{\boldsymbol{\tau}} & \hat{\boldsymbol{\alpha}}\end{array}\right]$

$$
\check{\mathbf{I}}=\left[\begin{array}{cc}
\tilde{\mathbf{I}}_{\mathrm{MP}} & \mathbf{J} \\
\mathbf{J}^{T} & \hat{\mathbf{I}}
\end{array}\right]
$$

where $\tilde{\mathbf{I}}_{\mathrm{MP}}$ is as given in Proposition 3

$$
\hat{\mathbf{I}}=\left[\begin{array}{ll}
\tilde{\mathbf{I}}_{\hat{\tau} \hat{\tau}} & \tilde{\mathbf{I}}_{\hat{\tau} \hat{\boldsymbol{\alpha}}} \\
\tilde{\mathbf{I}}_{\hat{\tau} \hat{\boldsymbol{\alpha}}}^{T} & \tilde{\mathbf{I}}_{\hat{\boldsymbol{\alpha}} \hat{\boldsymbol{\alpha}}}
\end{array}\right]
$$

and

$$
\mathbf{J}=\left[\begin{array}{ll}
\tilde{\mathbf{I}}_{\lambda \hat{\tau}} & \tilde{\mathbf{I}}_{\boldsymbol{\lambda}} \\
\tilde{\mathbf{I}}_{\tilde{\boldsymbol{\alpha}} \hat{\tau}} & \tilde{\mathbf{I}}_{\tilde{\tau} \hat{\alpha}} \\
\tilde{\mathbf{I}}_{\tilde{\boldsymbol{\alpha}} \hat{\tau}} & \tilde{\mathbf{I}}_{\tilde{\alpha} \hat{\alpha}}
\end{array}\right] .
$$

Since the multipath components in $\mathcal{L}-\tilde{\mathcal{L}}$ do not overlap with any of the multipath components in $\tilde{\mathcal{L}}$ [cf. Equation (29)-(32)], it can be shown from (53)-(55) that $\mathbf{J}=\mathbf{0}$. Therefore, $\breve{\mathbf{I}}$ in (56) is a block-diagonal matrix, which implies that $\left[\check{\mathbf{I}}^{-1}\right]_{K \times K}=$ $\tilde{\mathbf{I}}_{\mathrm{MP}}^{-1}$. Hence, the CRLB for $\boldsymbol{\lambda}$ can be obtained directly from $\widetilde{\mathbf{I}}_{\mathrm{MP}}$ in this case.

$$
\begin{gathered}
{\left[\mathbf{I}_{\tau \tau}\right]_{l_{1} l_{2}}= \begin{cases}\frac{\alpha_{l_{1}}^{2} \tilde{E}_{l_{1}}}{\sigma^{2}}, & l_{1}=l_{2} \\
\frac{\alpha_{l_{1}} \alpha_{l_{2}}}{\sigma^{2}} \tilde{R}_{l_{1} l_{2}}\left(\tau_{l_{1}}-\tau_{l_{2}}\right), & l_{1}, l_{2} \in \mathcal{L}-\{b\}, l_{1} \neq l_{2} \\
\frac{\alpha_{l} \alpha_{b}}{N \sigma^{2}} \sum_{k=0}^{N-1} \tilde{R}_{b l}\left(\tau_{b}+h_{k}(\boldsymbol{\lambda})-\tau_{l}\right), & l_{1}=b, l_{2}=l \neq b \text { or } l_{2}=b, l_{1}=l \neq b\end{cases} } \\
{\left[\mathbf{I}_{\boldsymbol{\alpha} \boldsymbol{\alpha}}\right]_{l_{1} l_{2}}= \begin{cases}\frac{E_{l_{1}}}{\sigma^{2}}, & l_{1}=l_{2} \\
\frac{1}{\sigma^{2}} R_{l_{1} l_{2}}\left(\tau_{l_{1}}-\tau_{l_{2}}\right), & l_{1}, l_{2} \in \mathcal{L}-\{b\}, l_{1} \neq l_{2} \\
\frac{1}{N \sigma^{2}} \sum_{k=0}^{N-1} R_{b l}\left(\tau_{b}+h_{k}(\boldsymbol{\lambda})-\tau_{l}\right), & l_{1}=b, l_{2}=l \neq b \text { or } l_{2}=b, l_{1}=l \neq b\end{cases} }
\end{gathered}
$$

$$
\left[\mathbf{I}_{\tau \boldsymbol{\alpha}}\right]_{l_{1} l_{2}}= \begin{cases}-\frac{\alpha_{l_{1}} \hat{E}_{l_{1}}}{\sigma^{2}}, & l_{1}=l_{2} \\ -\frac{\alpha_{l_{1}}}{\sigma^{2}} \hat{R}_{l_{1} l_{2}}\left(\tau_{l_{1}}-\tau_{l_{2}}\right), & l_{1} \neq l_{2}, l_{1}, l_{2} \in \mathcal{L}-\{b\} \\ -\frac{\alpha_{b}}{N \sigma^{2}} \sum_{k=0}^{N-1} \hat{R}_{b l_{2}}\left(\tau_{b}+h_{k}(\boldsymbol{\lambda})-\tau_{l_{2}}\right), & l_{1}=b, l_{2} \neq b \\ -\frac{\alpha_{l_{1}}}{N \sigma^{2}} \sum_{k=0}^{N-1} \hat{R}_{l_{1} b}\left(\tau_{l_{1}}-\tau_{b}-h_{k}(\boldsymbol{\lambda})\right), & l_{1} \neq b, l_{2}=b\end{cases}
$$




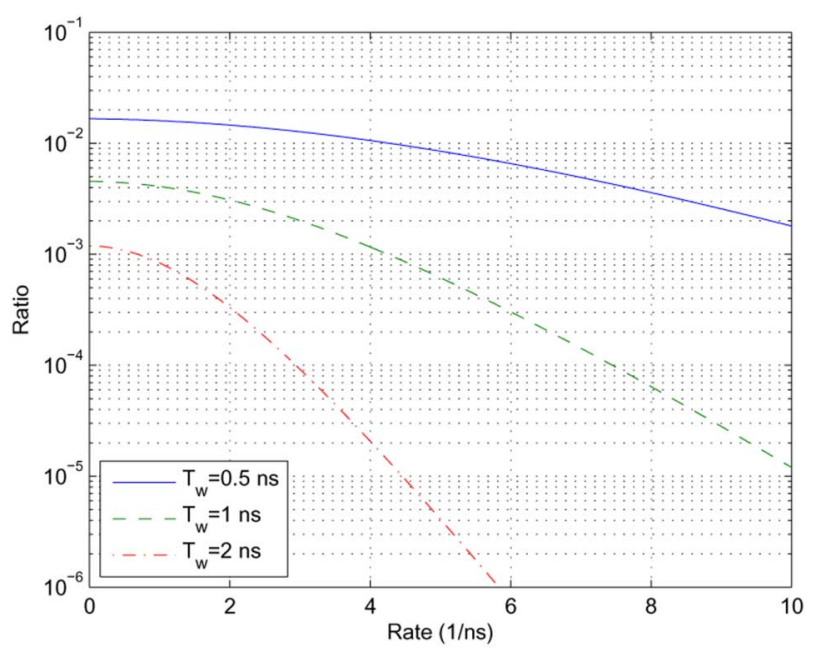

Fig. 11. Ratio between the second and the first term of (60) for $h_{M}=0.1 \mathrm{ns.}$

D. Approximate Independence of $\left\{C_{i}^{(\mathrm{u})}\right\}_{i=1}^{N_{L}^{(\mathrm{u})}}$ : First consider $C_{1}^{(\mathrm{u})}$ and $C_{2}^{(\mathrm{u})}$. The conditional probability of $C_{2}^{(\mathrm{u})}$ given $C_{1}^{(\mathrm{u})}$ can be expressed as

$$
\begin{aligned}
& P\left(C_{2}^{(\mathrm{u})} \mid C_{1}^{(\mathrm{u})}\right) \\
& =P\left(\tau_{b+2}-\tau_{b+1}<T_{\mathrm{w}}\right) \\
& \quad+P\left(\tau_{b+2}-\tau_{b+1} \geq T_{\mathrm{w}} \& \tau_{b+2}-\tau_{b}\right. \\
& \left.\quad<T_{\mathrm{w}}+h_{M} \mid \tau_{b+1}-\tau_{b}<T_{\mathrm{w}}+h_{M}\right)
\end{aligned}
$$

where the independence of intervals between different path arrivals is used. After some manipulation, it can be shown that

$$
\begin{aligned}
P\left(C_{2}^{(\mathrm{u})} \mid C_{1}^{(\mathrm{u})}\right)= & \left(1-e^{-\varphi T_{\mathrm{w}}}\right) \\
& +\frac{e^{-\varphi T_{\mathrm{w}}}-e^{-\varphi\left(T_{\mathrm{w}}+h_{M}\right)}\left(1+\varphi h_{M}\right)}{1-e^{-\varphi\left(T_{\mathrm{w}}+h_{M}\right)}}
\end{aligned}
$$

where the first term corresponds to $P\left(\tau_{b+2}-\tau_{b+1}<T_{\mathrm{w}}\right)$.

For practical values of the system parameters and for $h_{M} \ll$ $T_{\mathrm{w}}$, the ratio between the second term and the first term in (59) can be shown to be very small. For example, for $h_{M}=0.1 \mathrm{~ns}$, that ratio is plotted in Fig. 11 for various rate parameters of the Poisson process. Hence, it can be shown that

$$
P\left(C_{2}^{(\mathrm{u})} \mid C_{1}^{(\mathrm{u})}\right) \approx\left(1-e^{-\varphi T_{\mathrm{w}}}\right) \approx P\left(C_{2}^{(\mathrm{u})}\right)
$$

Similar analysis can also be performed for $P\left(C_{i}^{(\mathrm{u})} \mid C_{1}^{(\mathrm{u})} \cdots C_{i-1}^{(\mathrm{u})}\right)$ for $i>2$. Hence, the PMF of $Y^{\mathrm{u})}$ in (38) can be approximated as

$$
P\left(Y^{(\mathrm{u})}=n\right) \approx P\left(C_{1}^{(\mathrm{u})}\right) \prod_{j=2}^{n} P\left(C_{j}^{(\mathrm{u})}\right)\left(1-P\left(C_{n+1}^{(\mathrm{u})}\right)\right)
$$

from which (39) can be obtained. Similar analysis also applies to the PMF of $Y^{(1)}$.
E. Proof of Corollary 1: For $N_{L}^{(\mathrm{u})}=N_{L}^{(1)}=0$, there is no multipath component overlapping with the $b$ th component; i.e., $\tilde{\mathcal{L}}=\{b\}$ [see (33) and (34)]. Therefore, the reduced FIM can be obtained from Proposition 3 and Appendix B as

$$
\tilde{\mathbf{I}}_{\mathrm{MP}}=\left[\begin{array}{ll}
\mathbf{I}_{\boldsymbol{\lambda} \boldsymbol{\lambda}} & \mathbf{B} \\
\mathbf{B}^{T} & \mathbf{D}
\end{array}\right]
$$

where

$$
\mathbf{D}=\frac{1}{\sigma^{2}}\left[\begin{array}{cc}
\alpha_{b}^{2} \tilde{E}_{b} & -\alpha_{b} \hat{E}_{b} \\
-\alpha_{b} \hat{E}_{b} & E_{b}
\end{array}\right]
$$

and $\mathbf{B}$ is a $K \times 2$ matrix with its $i$ th row, given by

$$
[\mathbf{B}]_{i:}=\frac{\alpha_{b} \gamma_{i}}{N \sigma^{2}}\left[\begin{array}{ll}
\alpha_{b} \tilde{E}_{b} & \hat{E}_{b}
\end{array}\right]
$$

with $\gamma_{i}$ being given by (45).

From the formula for block matrix inversion [41], it can be shown that

$$
\left[\tilde{\mathbf{I}}_{\mathrm{MP}}^{-1}\right]_{K \times K}=\mathbf{I}_{\boldsymbol{\lambda} \boldsymbol{\lambda}}^{-1}+\mathbf{I}_{\boldsymbol{\lambda} \boldsymbol{\lambda}}^{-1} \mathbf{B}\left(\mathbf{D}-\mathbf{B}^{T} \mathbf{I}_{\boldsymbol{\lambda} \boldsymbol{\lambda}}^{-1} \mathbf{B}\right)^{-1} \mathbf{B}^{T} \mathbf{I}_{\boldsymbol{\lambda} \boldsymbol{\lambda}}^{-1} .
$$

Then, from the fact that $\mathbf{I}_{\boldsymbol{\lambda} \boldsymbol{\lambda}}=\alpha_{b}^{2} \mathbf{I}_{\mathrm{SP}}$, the expression in Corollary 1 can be obtained from (64)-(66), (11) and (12), after some manipulation.

F. Proof of Proposition 4: From the expression in (43), it is observed that

$$
\begin{aligned}
\lim _{N \rightarrow \infty} N^{2}\left[\mathbf{I}_{\mathrm{MP}}^{-1}\right]_{11} \\
=\frac{1}{\alpha_{b}^{2}} \lim _{N \rightarrow \infty} N^{2}\left[\mathbf{I}_{\mathrm{SP}}^{-1}\right]_{11} \\
\quad+\frac{\sigma^{2}}{\alpha_{b}^{2} \tilde{E}_{b}} \lim _{N \rightarrow \infty} \frac{\left(N\left[\boldsymbol{\Upsilon}^{-1}\right]_{1:} \boldsymbol{\gamma}\right)^{2}}{\left(1-\frac{1}{N} \boldsymbol{\gamma}^{T} \boldsymbol{\Upsilon}^{-1} \boldsymbol{\gamma}\right)}
\end{aligned}
$$

It can be shown, from (44) and (45), that the second term becomes zero ${ }^{19}$. Therefore, the result in the proposition follows directly from Proposition 1.

\section{REFERENCES}

[1] U. S. Federal Communications Commission FCC 02-48: First Report and Order

[2] S. Gezici, Z. Tian, G. B. Giannakis, H. Kobayashi, A. F. Molisch, H. V. Poor, and Z. Sahinoglu, "Localization via ultra-wideband radios," IEEE Signal Process. Mag. (Special Issue on Signal Processing for Positioning and Navigation with Applications to Communications), vol. 22, no. 4, pp. 70-84, Jul. 2005.

[3] S. Gezici, Z. Sahinoglu, H. Kobayashi, and H. V. Poor, H. Arslan, Z N. Chen, and M.-G. Di Benedetto, Eds., "Ultra wideband geolocation," in Ultra Wideband Wireless Communications, Oct. 2006.

[4] M. Z. Win and R. A. Scholtz, "Impluse radio: How it works," IEEE Commun. Lett., vol. 2, pp. 36-38, Feb. 1998.

[5] M. Z. Win, R. A. Scholtz, and L. W. Fullerton, "Time-hopping ssma techniques for impulse radio with an analog modulated data subcarrier," in Proc. IEEE 4th Int. Symp. Spread Spectrum Techniques \& Applications, Mainz, Germany, Sep. 1996, pp. 359-364.

[6] M. Z. Win and R. A. Scholtz, "Ultra-wide bandwidth time-hopping spread-spectrum impulse radio for wireless multiple-access communications," IEEE Trans. Commun., vol. 48, no. 4, pp. 679-691, Apr. 2000.

${ }^{19}$ The details are omitted due to the space limitation. 
[7] D. Cassioli, M. Z. Win, and A. F. Molisch, "The ultra-wide bandwidth indoor channel: From statistical model to simulations," IEEE J. Select. Areas Commun., vol. 20, no. 8, pp. 1247-1257, Aug. 2002.

[8] A. E. C. Tan and M. Y. W. Chia, "UWB radar transceiver and measurement for medical imaging," in Proc. IEEE Int. Workshop on Biomedical Circuits and Systems, Dec. 1-3, 2004, pp. S3.1 9-12.

[9] L. Xu, E. J. Bond, B. D. Van Veen, and S. C. Hagness, "An overview of ultra-wideband microwave imaging via space-time beamforming for early-stage breast-cancer detection," IEEE Antennas Propag. Mag., vol. 47, no. 1, pp. 19-34, Feb. 2005.

[10] A. Reisenzahn, T. Buchegger, D. Scherrer, S. Matzinger, S. Hantscher, and C. G. Diskus, "A ground penetrating UWB radar system," in Proc. 3rd Int. Conf. Ultrawideband and Ultrashort Impulse Signals, Sep. 2006, pp. 116-118.

[11] S. Hantscher, B. Praher, A. Reisenzahn, and C. G. Diskus, "Comparison of UWB target identification algorithms for through-wall imaging applications," in Proc. 3rd European Radar Conf., Sep. 2006, pp. 104-107.

[12] Y. Yunqiang and A. E. Fathy, "See-through-wall imaging using ultra wideband short-pulse radar system," in Proc. IEEE Antennas and Propagation Society Int. Symp., Jul. 3-8, , vol. 3B, pp. 334-337.

[13] S. E. Borek, "An overview of through the wall surveillance for homeland security," in 34th Proc. Applied Imagery and Pattern Recognition Workshop, Oct. 19-21, 2005.

[14] J. D. Taylor, Ed., Ultra-wideband Radar Technology. Boca Raton, FL: CRC Press, 2000.

[15] C. E. Cook and M. Bernfeld, Radar Signal: An Introduction to Theory and Application. New York: Academic, 1970.

[16] M. Nowogrodzki, D. D. Mawhinney, and H. F. Milgazo, "Noninvasive microwave instruments for the measurement of respiration and heart rates," in Proc. IEEE National Aerospace and Electronics Conf. (NAECON 1984), New York, May 21, 1984, vol. 2, pp. 958-960.

[17] O. B. Lubecke, P. W. Ong, and V. M. Lubecke, " $10 \mathrm{GHz}$ doppler radar sensing of respiration and heart movement," in Proc. IEEE 28th Аnnu. Northeast Bioengineering Conf., Philadelphia, PA, Apr. 20-21, 2002, pp. 55-56.

[18] S. Nag, H. Fluhler, and M. Barnes, "Preliminary interferometric images of moving targets obtained using a time-modulated ultra-wide band through-wall penetration radar," in Proc. IEEE Radar Conf., May 1-3, 2001, pp. 64-69.

[19] L. Huang and C. C. Ko, "Performance of maximum-likelihood channel estimator for UWB communications," IEEE Commun. Lett., vol. 8, no. 6, pp. 356-358, Jun. 2004.

[20] A.-L. Deleuze, C. L. Martret, P. Ciblat, and E. Serpedin, "Cramer-Rao bound for channel parameters in ultra-wide band based system," in Workshop on Signal Processing Advances in Wireless Communications (SPAWC04), Jul. 2004.

[21] J. Zhang, R. Kennedy, and T. Abhayapala, "Cramer-Rao lower bounds for the time delay estimation of UWB signals," in Proc. IEEE Int. Conf. Communications (ICC 2004), Paris, France, Jun. 20-24, 2004, vol. 6, pp. 3424-3428.

[22] V. Lottici, A. D'Andrea, and U. Mengali, "Channel estimation for ultra-wideband communications," IEEE J. Select. Areas Commun., vol. 20, no. 12, pp. 1638-1645, Dec. 2002.

[23] S. Venkatesh, C. R. Anderson, N. V. Rivera, and R. M. Buehrer, "Implementation and analysis of respiration-rate estimation using impulse-based UWB," in Proc. IEEE Military Communications Conf. (MILCOM 2005), Atlantic City, NJ, Oct. 17-20, 2005, vol. 5, pp. 3314-3320.

[24] E. M. Staderini, "UWB radars in medicine," IEEE Aerosp. Electron. Mag., vol. 17, no. 1, pp. 13-18, Jan. 2002.
[25] I. Y. Immoreev, S. Samkov, and T.-H. Tao, "Short-distance ultrawideband radars," IEEE Aerosp. Electron. Syst. Mag., vol. 20, no. 6, pp. 9-14, Jun. 2005.

[26] G. Ossberger, T. Buchegger, E. Schimback, A. Stelzer, and R. Weigel, "Non-invasive respiratory movement detection and monitoring of hidden humans using ultra wideband pulse radar," in Proc. IEEE Int. Workshop on Ultra Wideband Systems (IWUWBS 2004), Piscataway, NJ, May 18-21, 2004, pp. 395-399.

[27] Y. Chen, E. Gunawan, K. S. Low, Y. Kim, C. B. Soh, A. R. Leyman, and L. L. Thi, "Non-invasive respiration rate estimation using ultra-wideband distributed cognitive radar system," in Proc. 28th IEEE EMBS Annu. Int. Conf., Aug.-Sep. 30-3, 2006, pp. 920-923.

[28] M. A. Richards, Fundamentals of Radar Signal Processing, 1st ed ed. New York: McGraw-Hill, 2005.

[29] H. V. Poor, An Introduction to Signal Detection and Estimation, 2nd ed. New York: Springer-Verlag, 1994.

[30] J. Caffery, Jr., Wireless Location in CDMA Cellular Radio Systems. Boston, MA: Kluwer, 2000.

[31] Y. Qi, H. Kobayashi, and H. Suda, "On time-of-arrival positioning in a multipath environment," IEEE Trans. Veh. Technol., vol. 55, no. 5, pp. 1516-1526, Sep. 2006.

[32] Y. Qi, "Wireless Geolocation in a Non-Line-of-Sight Environment," Ph.D. dissertation, Princeton Univ., Princeton, NJ, 2003.

[33] S. M. Kay, Fundamentals of Statistical Signal Processing, Volume I: Estimation Theory. Englewood Cliffs, NJ: Prentice-Hall, 1993.

[34] A. A. M. Saleh and R. Valenzuela, "A statistical model for indoor multipath propagation," IEEE J. Select. Areas Commun., vol. SAC-5, no. 2, pp. 128-137, Feb. 1987.

[35] J. Foerster et al., Channel Modeling Sub-Committee Rep. Final 2003, IEEE, Tech. Rep.P802.15-02/490r1-SG3a.

[36] A. F. Molisch et al., "IEEE 802.15.4a channel model-Final report," IEEE P802.15-04/662rO, Nov. 2004.

[37] Y. Xu, W. F. Feng, J. Y. Hao, and H. K. Hwang, "Adaptive radar clutter suppression," in Proc. OCEANS 2001 Conf., MTS/IEEE Conf. and Exhib., Nov. 5-8, 2001, vol. 2, pp. 762-768.

[38] M. I. Skolnik, Introduction to Radar Systems, 2nd ed. New York: McGraw-Hill, 1980.

[39] F. Ramirez-Mireles and R. A. Scholtz, "Multiple-access performance limits with time hopping and pulse-position modulation," in Proc. IEEE Military Communications Conf. (MILCOM '98), Boston, MA, Oct. 1998, vol. 2, pp. 529-533.

[40] I. S. Gradshteyn and I. M. Ryzhik, Table of Integrals, Series, and Products, 6th ed. SanDiego: Academic Press, 2000.

[41] G. H. Gloub and C. F. Van Loan, Matrix Computations, 3rd ed. Baltimore, MD: The Johns Hopkins Univ. Press, 1996.

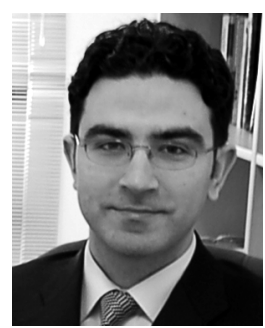

Sinan Gezici (M'02) received the B.S. degree from Bilkent University, Ankara, Turkey, in 2001, and the $\mathrm{Ph} . \mathrm{D}$. degree in electrical engineering from Princeton University, Princeton, NJ, in 2006.

From April 2006 to January 2007, he was a Visiting Member of Technical Staff at Mitsubishi Electric Research Laboratories, Cambridge, MA. Since February 2007, he has been an Assistant Professor in the Department of Electrical and Electronics Engineering at Bilkent University. His research interests are in the areas of signal detection, estimation and optimization theory, and their applications to wireless communications and localization systems. Currently, he has a particular interest in ultrawideband systems for communications and sensing applications. 